Bull. Fac.Agric.,Cairo Univ., 63:188-200 (2012).

\title{
SELECTION OF LOCAL OKRA (Abelmoschus esculentus L.) GENOTYPES FOR STABILITY UNDER SALINE CONDITIONS
}

(Received:6.3.2012)

\author{
By \\ H. H. Hamed and M. R. Hafiz* \\ Horticulture Research Institute, Agriculture Research Center, Giza, Egypt. \\ and $*$ Plant Production Department, Desert Research Center,Cairo
}

\begin{abstract}
This investigation aimed to observe genotypic stability (with respect to pods yield) of thirteen local okra genotypes across three locations: Kaha Horticulture Research Station, Kaluobia Governorate, Experimental Farm of the Desert Research Center at Ras Sudr, South Sinai Governorate location 1,2 (both salinity condition). In addition to group, the genotypes having similar response pattern over all environments. Moreover, studying the effect of salinity conditions on plant growth performance. Multienvironmental trials (MET), generally, have significant main effects and significant multiplicative genotype $\mathrm{x}$ environment interaction effect. AMMI (Additive main effect and multiplicative interaction analysis) offers a more appropriate statistical analysis to deal with such situations, compared to traditional methods like ANOVA, PCA and Linear regression.

The results showed that (I) the obtained results satisfied one of the breeder's goals for selecting the best-suited genotype for cultivation in a wide salinity range of environments; (II) the analysis of variance of thirteen local okra genotypes in three locations (Kaha, Ras sudr 1 and Ras sudr 2) shows that genotype $(\mathrm{G})$, environment $(\mathrm{E})$ and their interaction were significante $(\mathrm{P}<0.01)$ for genotype; (III) the AMMI model was very effective for studying GEI interaction, the first bilinear AMMI (IPCA1) model terms accounted for 71.268\%; (IV) no genotype has superiority performance in under all studied environments; although, the biplot shows that the genotypes BG9, BG6, BR27 and BR20 are best-suited for cultivation in a wide range of environments; (V) the salt stress has affected the Okra plant growth and development.
\end{abstract}

Key words: AMMI model, biplot, Okra, salinity, yield stability.

\section{INTRODUCTION}

Excess amount of salt in the soil adversely affects plant growth and development. Nearly $20 \%$ of the world's cultivated area and nearly half of the world's irrigated lands are affected by salinity (Ashraf 1994). For improving the salt stress tolerance of crop varieties by plant breeding, it is necessary to identify donor genotypes that have proven tolerance to salt stress during all the growth stages. Genotype $\mathrm{x}$ environment $(\mathrm{G} \times \mathrm{E})$ interaction plays a major role in evaluation of genotypes under different environments (salinity stress) to identify genotypes suitable to different stresses (Munns and James 2003). Genotype - environment interaction (GEI) is the differential response of genotypes to changing environmental conditions.

An ideal variety should have a high mean yield combined with a low degree of fluctuation, when grown over diverse environments. Two main contrasting concepts of stability are distinguished: "static" (Type 1) and "dynamic" (Type 2) (Lin et al., 1986; Becker and Leon, 1988). For static stability, the best genotype tends to maintain a constant yield across environments. Dynamic stability implies for a stable genotype a yield response in each environment that is always parallel to the mean response of the tested genotypes, i.e. zero GEI (Annicchiarico, 2002). Analyzing of GEI for varieties can reduce errors in the breeding process as the selection in one condition cannot provide advantage in others (Lin et al., 1986). The analysis of variance (ANOVA) provides no insight into the particular pattern of the underlying interaction (Gauch and Zobel 1988, Zobel et al., 1988); while, the Linear regression model of Eberhart and Russell, 1966 is most frequently used for GXE interaction study and in this model a stable genotype should have low deviation from regression $\left(S^{2}{ }_{d}\right)$. So, many genotypes having very high yield potential often get rejected due to high $\mathrm{S}_{\mathrm{d}}^{2}$ over the range of 
environments. Thus, a genotype showing high positive interaction at certain environments and negative interaction at others is likely to show high $S_{d}^{2}$ and would be classified as unstable. The LR model does not provide for critical analysis of interaction of genotypes in specific environments and does not help in identifying promising genotypes to take advantage of their high positive interaction with the agro-ecological conditions of specific locations or specific agro-management conditions like early or late sowing, high or low fertility, rained or irrigated etc. (Misra et al., 2009). On the other hand, the AMMI analysis model is additive and effectively describes the main (additive) effects, while the interaction (residual from the additive model) is non additive and requires other techniques, such as principal component analysis (PCA) to identity interaction patterns. Thus, ANOVA and PCA models combined to constitute the additive Main - effect and Multiplicative interaction (AMMI) model (Gauch and Zobel 1988, Zobel et al., 1988). The AMMI model is, therefore, a hybrid statistical model incorporating both ANOVA (for additive component) and PCA (for multiplicative component) for analyzing two-way (genotype - by -environment) data structure. The model has, in recent past, been recommended for statistical analysis of yield trials, and was preferred over other customary statistical analyses, such as ordinary ANOVA, principal component analysis and linear regression analysis (Gauch 1988, Zobel et al., 1988). The results of AMMI analysis are useful in supporting breeding program decisions such as specific adaptation and selection of environment (Gauch and Zobel, 1997). Usually, the results of AMMI analysis shown in common graphs are called biplot (Gabriel, 1971). The biplot shows both the genotypes and the environment value and relationships using singular vector technique (Eckart and Young 1936 C.A. Tarakanovas and Ruzgas, 2006).

The present study was initiated to achieve the following objectives:

* To observe genotypic stability (with respect to pods yield) of 13 local Okra genotypes across 3 locations (Two of them with the properties of salinity) in Egypt.

* To group the genotypes having similar response pattern over all environments.

\section{MATERIALS AND METHODS}

Thirteen accessions /genotypes of local okra, "Balady" green (BG) characterized by semi-long stemmed $(106 \mathrm{~cm})$; green, moderate spiny pods, and "Balady" red (BR) characterized by short stemmed $(85 \mathrm{~cm})$; red-cornered, smooth pods. These thirteen genotypes were obtained from previous selection program (Hamed et al., 2003). The multi locational evaluation trials were carried out during cropping seasons 2009 - 2010 conducted at different three locations in Egypt, with respect to average salinity (whether soil and irrigation water) (Table 1) and Table (2), respectively.

The genotypes namely; BG4, BG14, BG6, BG21, BG7, BG12, BG9, BR21, BR16, BR20, BR15, BR27 and BR4.

Data were recorded on the following characters:

1- Pod weight (g) 2- Yield per plant (g).

3- Plant height $(\mathrm{cm})$. 4- No. of branches 4 plant. 5- Pod diameter $(\mathrm{cm})$ 6- Pod length $(\mathrm{cm})$.

7- No. of total pods/ plant.

8- Germination percentage 9- Root length $(\mathrm{cm})$.

10- Shoot length $(\mathrm{cm})$

11- $\mathrm{Na}^{+} 10 \mathrm{n}$ concentration $(\mathrm{mM} / \mathrm{L})$

$12-\mathrm{K}^{+}$ion concentration $(\mathrm{mM} / \mathrm{L})$.

$13-\mathrm{Na}^{+} / \mathrm{K}^{+}$ratio concentration (\%)

Measurements of root length, shoot length and chemical analyses were conducted on seedling stage (after about 21 days of planting in the field). Seeds of the used genotypes were sown in seedling trays on 15 March. After sowing, the seedlings (15 days old), of each genotype were transplanted in the field. The area of the plot was divided into 6 ridges. Each ridge was $70 \mathrm{~cm}$ wide and $3.5 \mathrm{~m}$ long. Seedlings were transplanted on only one side of the ridge at distances of $50 \mathrm{~cm}$. All experimental units received identical care regarding cultivation, manuring, fertilization, irrigation, pest control, and all other agricultural practices; that were performed as commonly followed in the experiment districts. The $\mathrm{Na}^{+}$and $\mathrm{K}^{+}$ion contents in the sap were measured with a flame photometer according to Chapman and Pratt (1961).

\section{Statistical analysis}

Layout of all the experiments was Randomized Complete Block Design (RCBD) with three replications. To determine the effects of genotype $\mathrm{x}$ environment interaction on yields, the data were subjected to Additive Main effects and Multiplicative Interaction (AMMI) analysis using IRRISTAT package (IRRI, 2003) and the biplot drawn by placing both genotype and environment means on the $\mathrm{x}$ - axis or abscissa and the respective eigenvectors or scores (IPCAI) on the y-axis or model is:

The AMMI model is:

$\mathrm{Y}_{\text {ger }}=\mu+\propto \mathrm{g}+\beta_{\mathrm{e}}+\Sigma_{\mathrm{n}} \lambda_{\mathrm{n}} \gamma_{\mathrm{gn}} \delta_{\mathrm{en}}+\rho_{\mathrm{ge}}+\mathrm{C}_{\mathrm{ger}}$ 
Wherein $\mathrm{Y}_{\text {ger }}=$ yield of genotype "g" in environment "e" for replicate $\mathrm{r} ; \mu=$ grand mean; $\propto \mathrm{g}=$ mean deviation of the genotype $\mathrm{g}$ [genotype mean minus grand mean] and $\beta_{\mathrm{e}}=$ mean deviation of environmental mean; $\lambda_{n}=$ the singular value for IPCA axis $\mathrm{n} ; \gamma_{\mathrm{gn}}=$ the genotype $\mathrm{g}$ eigenvector value for IPCA axis $n$; $\delta_{\text {en }}=$ the environment $\mathrm{e}$ eigenvector value for IPCA axis $\mathrm{n} ; \rho_{\mathrm{ge}}=$ the residual and $\mathrm{C}_{\mathrm{ger}}=$ the error. The means were separated using Fisher's protected least significance difference test (LSD) at $\mathrm{P}=0.01$.

\section{RESULTS AND DISCUSSION \\ 3.1. Stability and adaptation of local Okra genotypes.}

AMMI analysis (Zobel et al., 1988 and Purchase, 1997) gives estimate of total G x E interaction effect of each genotype and also further partitioned it into interaction effects due to individual environments. Low $\mathrm{G} \times \mathrm{E}$ interaction of a genotype indicates stability of the genotype over the range of environments. A genotype showing high positive interaction in an environment obviously has the ability to exploit the agroecological or agro-management conditions of the specific environment and is therefore best suited to that environment. AMMI analysis permits estimation of interaction effect of a genotype in each environment and it helps to identify genotypes best suited for specific environmental conditions. Though analysis of $\mathrm{G} \times \mathrm{E}$ interaction of multilocation yield data in AMMI model have been reported by Vijaykumar et al., 2001; Mahalingam et al., 2006; Naveed et al., 2007; Das et al., 2009; Mohamadi et al., 2007; Shinde et al., 2002 and Hariprasanna et al., 2008. All those researchers stressed the usefulness of AMMI analysis for selection of promising genotypes for specific locations or environmental conditions.

The AMMI analysis of variance for pod yield (g\plant) (Table 3) indicated that genotypes, environments and $G \times x$ interaction were significantly different $(\mathrm{P}<0.01)$. The AMMI model supplied on adequate fit to the data as first Interaction Principle Component Axis (IPCA) was significant $(\mathrm{P}<0.01)$. The sum of squares for genotypes, environments and IPCAI provided $97.333 \%(14.872 \%+75.952 \%+6.539 \%)$ of total sum of squares indicating that AMMI model effectively partitioned total sum of squares (Siddiq, 1968). The main effects of $\mathrm{G}$ and $\mathrm{E}$ accounted for 14.872 and $75.952 \%$, respectively and $\mathrm{G} \times \mathrm{E}$ interaction accounted for $9.175 \%$ of the total variation in $\mathrm{G} \times \mathrm{E}$ interaction was further partitioned into IPCA 1 and IPCA 2, of which
IPCA I component was significant and accounted for $71.268 \%$ of the total $\mathrm{G} \times \mathrm{E}$ interaction sum of squares and used 13 of the total 24 available in the interaction, they were significant at $\mathrm{P}<0.01$. The obtained data confirm adequacy to the AMMI model. This made it possible to construct the biplot and calculate genotypes and environments effects (Yan and Hunt, 2001). The interaction principle component Axes (IPCA) scores of a genotype in the AMMI analysis indicate the stability of a genotype across environments. The closer the IPCA scores are to Zero, the more stable the genotypes are across their testing environments. Basically, these biplots belong to two types: AMMI 1 and AMMI 2 (Carbonell et al., 2004). IN AMMI 1, the genotype and environment means are plotted on the abscissa, and the IPCA scores for the same genotypes and environments, on the ordinate. For interpretation of the scores of the IPCA 1 are observed; scores close to zero are characteristic of genotypes and environments, which contribute little to the interaction, that is, they are stable.

Table (4) shows effects of genotypes and locations values from the additive genotype $\mathrm{x}$ environment model. The large differences of effect on both genotypes and on environments were observed. Environments Kaha (-5.871 glplant) and Ras Sudr 3 (-0.877 glplant) have the main significant negative pod yield effects. The genotypes BG6 (1.893 g/plant), BR27 (1.637 g/plant) BR15 (1.538 g/plant) and BR4 (0.653 $\mathrm{g} /$ plant) had a positive pod yield (g/plant) significant effect. The majority of the local Okra genotypes had a small not insignificant main negative or positive effect. Thus, many of these genotypes showed differential performance under different planting conditions. In Figure (1), the IPCAI scores for both the genotypes (number and environments (upper case) were plotted against the pod yield for the genotypes and the environments, respectively. We can clearly see the association between genotypes and the environments plotting on the same graph. The IPCA scores of a genotype in the AMMI analysis are an indication of the adaptability over environments.

The graph space of Fig. 1 is divided into 4 quadrants from lower yielding environments in Quadrants 1 and 4 to high yielding in quadrants 2 and 3. The biplot shows not only the average yield of a genotype but also how it is achieved. The genotypes BG 6 (no. 3), BR 27 (no. 12), BR 20 (no. 10) and BG 9 (no. 7) posed in quadrant 2 and 3 show that they have good adaptation to a wide 
Table (1): Soil chemical analysis of each experimental location.

\begin{tabular}{|c|c|c|c|c|c|c|c|c|c|c|c|c|c|c|c|c|c|c|c|}
\hline \multirow{2}{*}{ 泀 } & \multirow{2}{*}{ 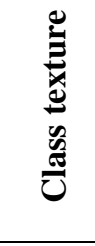 } & \multirow{2}{*}{ 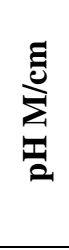 } & \multirow{2}{*}{ 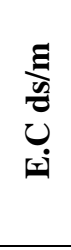 } & \multirow{2}{*}{$\underbrace{\infty}_{\tilde{\sigma}}$} & \multicolumn{4}{|c|}{$\begin{array}{c}\text { Soluble cations } \\
(\mathrm{M} / \mathrm{L})\end{array}$} & \multicolumn{4}{|c|}{$\begin{array}{c}\text { Soluble anions } \\
(\mathrm{M} / \mathrm{L})\end{array}$} & \multicolumn{3}{|c|}{$\begin{array}{l}\text { Macro elements } \\
\quad(\text { ppm })\end{array}$} & \multicolumn{4}{|c|}{$\begin{array}{l}\text { Micro elements } \\
\quad(\text { ppm) }\end{array}$} \\
\hline & & & & & T & $\stackrel{\sim}{+\infty}$ & $\stackrel{+}{+}^{\mathbf{Z}}$ & \pm & లి & 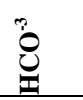 & 艺 & O̊ & Z & A & 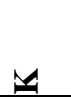 & ב & $\tilde{E}$ & $\tilde{\text { กี }}$ & $\Sigma$ \\
\hline KAHA & Loam & 8.4 & 0.39 & 3.6 & 1.0 & 0.65 & 2.19 & 0.48 & - & 1.9 & $\begin{array}{l}0.9 \\
\end{array}$ & 1.5 & 38 & 30 & 5.58 & 4.1 & 2.8 & 1.75 & 2.7 \\
\hline $\begin{array}{c}\text { Ras } \\
\text { Sudr1 }\end{array}$ & $\begin{array}{c}\text { Sandy } \\
\text { loam }\end{array}$ & 7.7 & 4.77 & 54.73 & 24.00 & 11.00 & 10.53 & 2.18 & - & 6.00 & 31.20 & 10.50 & - & - & 2.18 & - & - & - & - \\
\hline $\begin{array}{c}\text { Ras } \\
\text { Sudr2 }\end{array}$ & $\begin{array}{c}\text { Sandy } \\
\text { loam }\end{array}$ & 8.22 & 8.03 & 56.99 & 19.3 & 2.31 & 19.9 & 0.75 & - & 0.72 & 27.8 & 12.2 & 1.39 & - & 0.75 & - & - & - & 1.02 \\
\hline
\end{tabular}

*KAHA: Kaha Horticulture Research Station (Kaluobia Governorate, Egypt. (E1)

Ras Sudr1: Experimental Farm of the Desert Research Center at Ras Sudr, South Sinai, Egypt. (E2)

Ras Sudr2: Experimental Farm of the Desert Research Center at Ras Sudr, South Sinai, Egypt. (E3)

Table (2): Water chemical analysis of each experimental location.

\begin{tabular}{|c|c|c|c|c|c|c|c|c|c|c|c|c|c|c|c|c|c|c|}
\hline \multirow{2}{*}{ 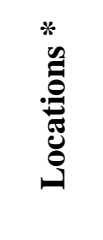 } & \multirow{2}{*}{ 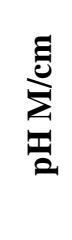 } & \multirow{2}{*}{ 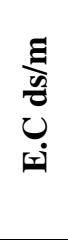 } & \multirow{2}{*}{ రీ } & \multicolumn{4}{|c|}{$\begin{array}{c}\text { Soluble cations } \\
\text { (M/L) }\end{array}$} & \multicolumn{4}{|c|}{$\begin{array}{c}\text { Soluble anions } \\
(\mathrm{M} / \mathrm{L})\end{array}$} & \multicolumn{3}{|c|}{$\begin{array}{l}\text { Macro elements } \\
\quad(\text { ppm })\end{array}$} & \multicolumn{4}{|c|}{$\begin{array}{c}\text { Micro elements } \\
(\text { ppm) }\end{array}$} \\
\hline & & & & $\stackrel{+}{\text { ? }}$ & $\sum^{+\infty}$ & ${ }^{+}$ & \pm & ชิ & 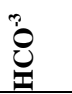 & 艺 & $\overbrace{}^{1}$ & $\mathbf{Z}$ & $A$ & 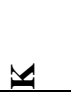 & 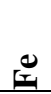 & E & งี & $\Sigma$ \\
\hline KAHA & $7 . .90$ & 0.37 & - & $1 . .55$ & 0.76 & 1.44 & 1.18 & - & 1.82 & 1.86 & 0.98 & - & - & 1.18 & - & - & - & - \\
\hline $\begin{array}{c}\text { Ras } \\
\text { Sudr1 }\end{array}$ & 8.40 & 5.47 & - & 23.65 & 19.18 & 56.66 & 0.51 & - & 2.50 & 16.22 & 81.33 & - & - & 0.51 & - & - & - & - \\
\hline $\begin{array}{c}\text { Ras } \\
\text { Sudr2 }\end{array}$ & 7.89 & 7.20 & - & 21.80 & 12.41 & 37.1 & 0.48 & - & 2.83 & 47.61 & 19.68 & - & - & 0.48 & - & - & - & - \\
\hline
\end{tabular}

*KAHA: Kaha Horticulture Research Station (Kaluobia Governorate, Egypt. (E1)

Ras Sudr1: Experimental Farm of the Desert Research Center at Ras surd, South Sinai, Egypt. (E2)

Ras Sudr2: Experimental Farm of the Desert Research Center at Ras surd, South Sinai, Egypt. (E3) 


\begin{tabular}{|c|c|c|c|c|c|c|}
\hline SOURCE & D.F. & S.S. & $\%$ of G-E SS & M.S. & P. & $\begin{array}{c}\% \text { of GxE } \\
\text { InteractionSS }\end{array}$ \\
\hline GENOTYPES & 12 & 14844.5 & $\% 14.872$ & 1237.04 & $* *$ & \\
\hline LOCATIONS & 2 & 75810.5 & $\% 85.952$ & 37905.3 & $* *$ & \\
\hline GX L & 24 & 9157.85 & $\% 9.175$ & 381.577 & $* *$ & \\
\hline IPCA 1 $^{\mathrm{a}}$ & 13 & 6526.59 & $\% 6.539$ & 502.045 & $* *$ & $\% 71.268$ \\
\hline IPCA 2 & 11 & 2631.26 & $\% 2.636$ & 239.205 & & $\% 28.732$ \\
\hline RESIDUAL & - & & & & & - \\
\hline TOTAL & 38 & 99812.9 & & & & \\
\hline
\end{tabular}

\begin{tabular}{|c|c|c|c|c|}
\hline \multicolumn{5}{|c|}{$\begin{array}{l}\text { Table (4): Interaction (additive) effects and multiplicative scores of local } \\
\text { okra genotypes for pod yield (g/ plant) in three environmental } \\
\text { conditions. }\end{array}$} \\
\hline \multirow{2}{*}{ Genotype location } & \multicolumn{3}{|c|}{ Environmental conditions } & \multirow{2}{*}{$\begin{array}{l}\text { Genotype } \\
\text { effects }\end{array}$} \\
\hline & E1 & E2 & E3 & \\
\hline BG4 & -1.273 & $\begin{array}{l}-0.834 \\
\end{array}$ & 2.106 & -3.412 \\
\hline BG14 & -4.366 & -2.860 & 7.226 & -1.439 \\
\hline BG6 & 2.575 & 1.687 & -4.262 & $1.893^{\text {क*** }}$ \\
\hline BG21 & -1.627 & -1.065 & 2.692 & -0.290 \\
\hline BG7 & 5.371 & 3.517 & -8.888 & 1.713 \\
\hline BG12 & 4.877 & 3.194 & -8.071 & -2.819 \\
\hline BG9 & 13.15 & 8.615 & -21.77 & -2.569 \\
\hline BR21 & -1.868 & -1.223 & 3.091 & -2.109 \\
\hline BR16 & -4.471 & -2.928 & 7.399 & -0.749 \\
\hline BR20 & 5.774 & 3.782 & -9.556 & 5.954 \\
\hline BR15 & -14.380 & -9.418 & 23.80 & $1.538^{* * * *}$ \\
\hline BR27 & 5.361 & 3.511 & -8.873 & $1.637^{\text {***\% }}$ \\
\hline BR4 & -9.129 & -5.978 & 15.11 & $\mathbf{0 . 6 5 3} 3^{\text {**ak }}$ \\
\hline Environment effects & $-5.871^{* * * *}$ & 6.749 & $-0.877^{* * * *}$ & \\
\hline \multicolumn{5}{|c|}{ 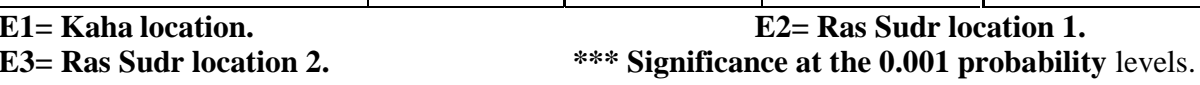 } \\
\hline
\end{tabular}

range of environments. Genotypes located near the plot origin were less responsive than the vertex genotypes. Considering only the IPCA 1 scores it became clear that the Genotype BG 9 (no. 7) was the most stable genotype, it was well adapted to high yielding environments that are more favorable with respect to the test sites, Kaha (E1) location was most discrimining as indicated by the longest distance between its marker and the origin, the length of a genotype vectors reflects the amount of interaction for that genotype. Thus, according to Fig. 1, most genetic environment interaction (GEI) is due to the fact that the genotype BR 20 (no. 10) has pod yield moderate average and large IPCA scores value in the trail. As a result, this genotype is most suitable for poor environments.

Fig. (2) gives the AMMI II biplot for yield. The IPCAI component accounted for $71.26 \%$ of GXE interaction, while IPCA 2 accounted for only $28.73 \%$ (Table 3). Distribution of genotype points in the AMMI II biplot revealed that the genotypes BG 21(no.4), BR 16 (no.9) and BG minimal interaction of these genotypes with environments. 


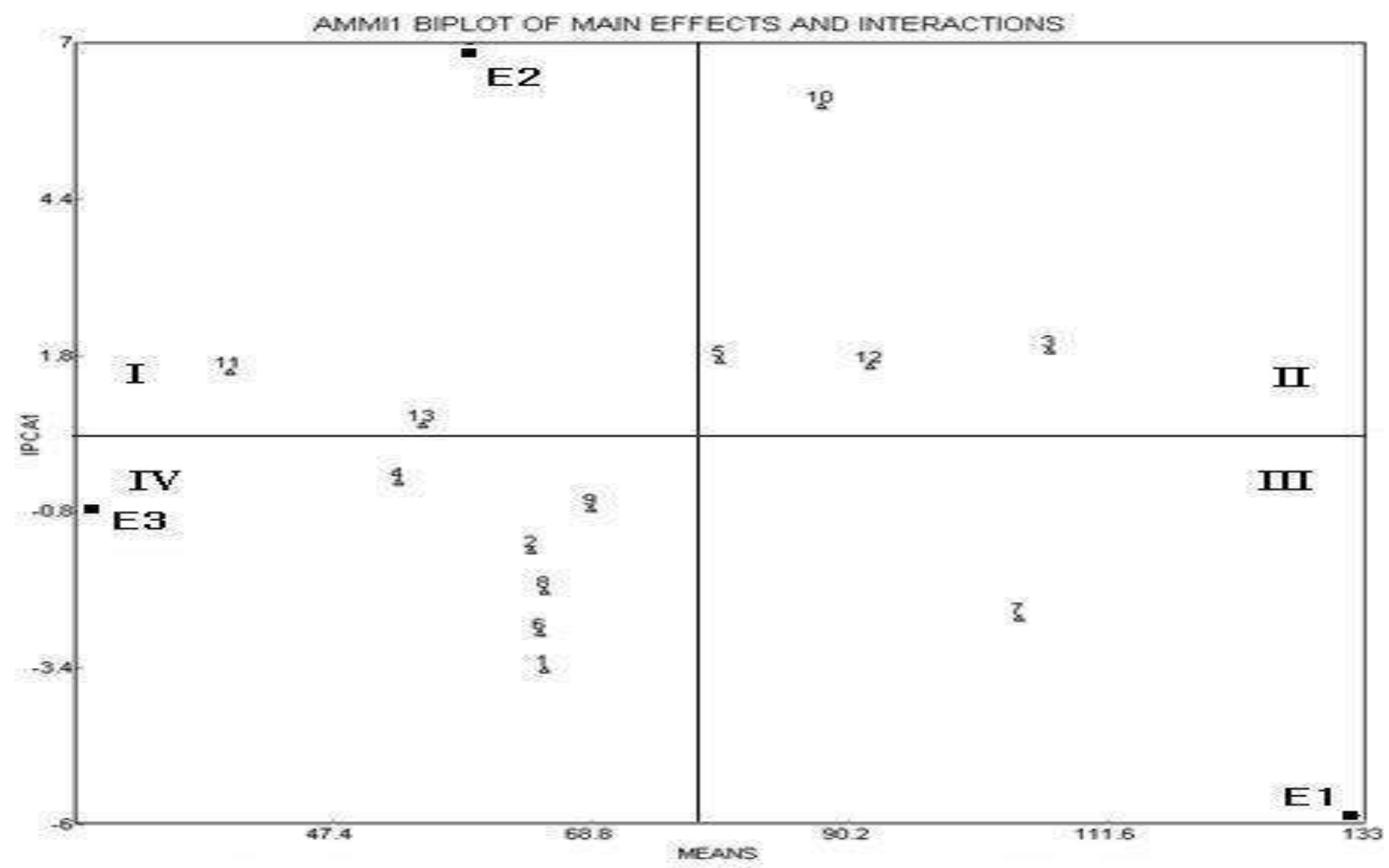

Fig.( 1): AMMI I biplot of main effects and GxE interaction of 13 local Okra genotypes at three locations of Egypt. BG4(no.1), BG14(no.2), BG6(no.3), BG21(no.4), BG7(no.5), BG12(no.6), BG9(no.7), BR21(no.8), BR16(no.9), BR20(no.10), BR15(no.11), BR27(no.12) and BR4(no.13). E1=Kaha location 1, E2=Ras Sudr location 1, E3=Ras Sudr location 2.

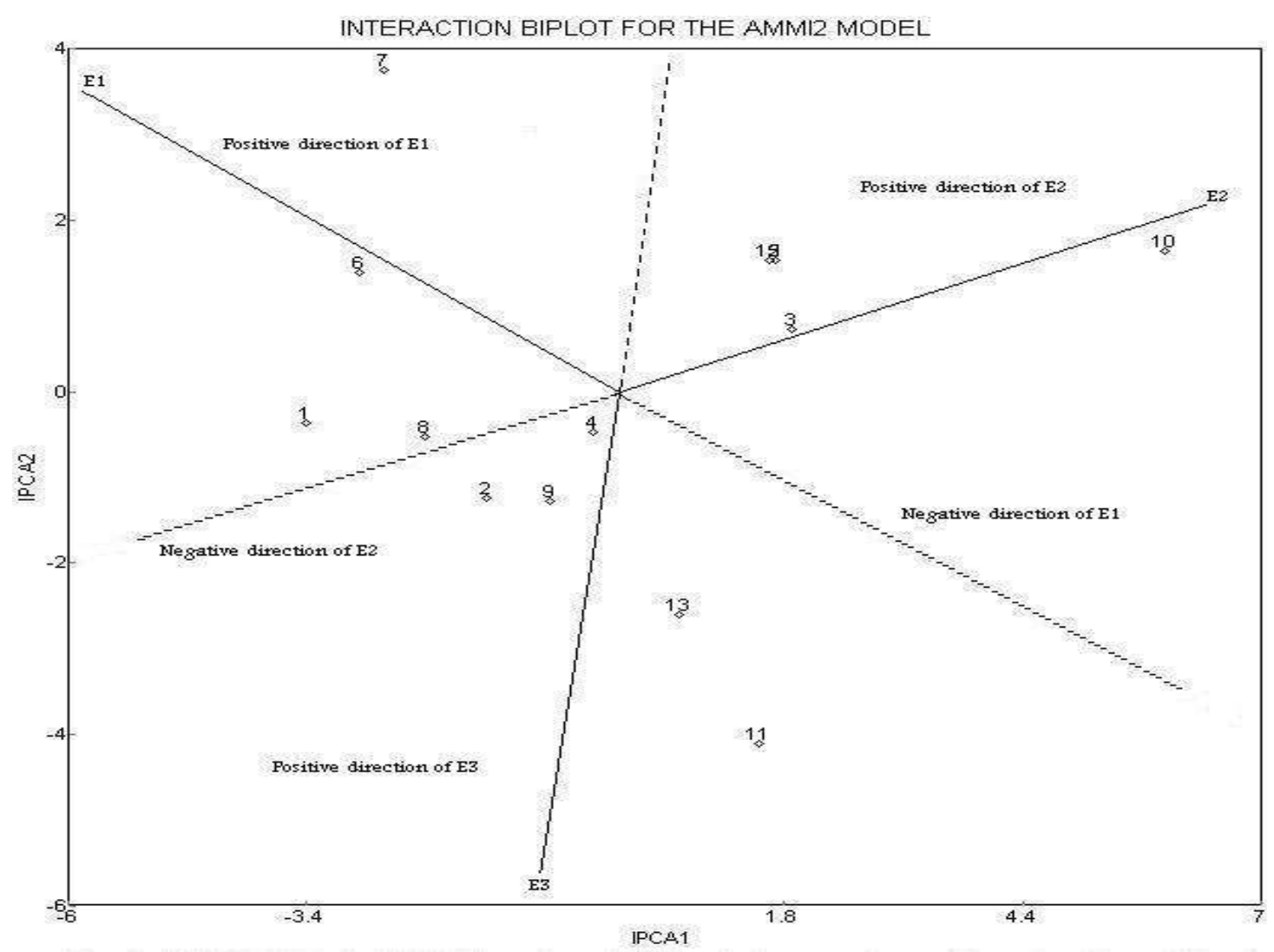

Fig. (2): AMMI II biplot of GxE interaction of 13 local Okra genotypes at three locations of Egypt. BG4(no.1), BG14(no.2), BG6(no.3), BG21(no.4), BG7(no.5), BG12(no.6), BG9(no.7), BR21(no.8), BR16(no.9), BR20(no.10), BR15(no.11), BR27(no.12) and BR4(no.13). E1=Kaha location 1, E2=Ras Sudr location 1, E3=Ras Sudr location 2. 


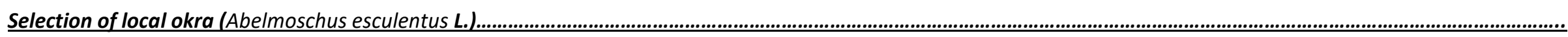

\begin{tabular}{|c|c|c|c|c|c|c|c|c|c|c|c|c|c|c|c|c|c|c|c|c|}
\hline & \multicolumn{4}{|c|}{ Pod weight } & \multicolumn{4}{|c|}{ yield per plant } & \multicolumn{4}{|c|}{ Plant height } & \multicolumn{4}{|c|}{ No. of branches/plant } & \multicolumn{4}{|c|}{ Pod diameter } \\
\hline & E1 & E2 & E3 & GM & E1 & E2 & E3 & GM & E1 & E2 & E3 & GM & E1 & E2 & E3 & GM & E1 & E2 & E3 & GM \\
\hline BG4 & $\begin{array}{c}4.985 \\
\pm 0.091\end{array}$ & $\begin{array}{c}3.640 \\
\pm 0.034\end{array}$ & $\begin{array}{c}2.667 \\
\pm 0.050\end{array}$ & $\begin{array}{c}3.76 \\
\mathrm{~cd}\end{array}$ & $\begin{array}{r}143.2 \\
\pm 17.2\end{array}$ & $\begin{array}{l}27.31 \\
\pm 6.86\end{array}$ & $\begin{array}{l}24.26 \\
\pm 3.52\end{array}$ & $\begin{array}{c}64.93 \\
\mathrm{de}\end{array}$ & $\begin{array}{r}92.67 \\
\pm 4.67\end{array}$ & $\begin{array}{l}69.00 \\
\pm 1.00\end{array}$ & $\begin{array}{l}41.00 \\
\pm 0.57\end{array}$ & $\begin{array}{c}67.55 \\
\text { bcd }\end{array}$ & $\begin{array}{l}3.667 \\
\pm 0.66\end{array}$ & $\begin{array}{l}3.667 \\
\pm 0.33\end{array}$ & $\begin{array}{l}3.667 \\
\pm 0.33\end{array}$ & $\begin{array}{c}3.66 \\
a b\end{array}$ & $\begin{array}{l}1.539 \\
\pm 0.06\end{array}$ & $\begin{array}{r}1.539 \\
\pm 0.11\end{array}$ & $\begin{array}{l}1.196 \\
\pm 0.04\end{array}$ & $\begin{array}{c}1.424 \\
\text { bc }\end{array}$ \\
\hline BG14 & $\begin{array}{c}5.093 \\
\pm 0.184\end{array}$ & $\begin{array}{c}4.091 \\
\pm 0.110\end{array}$ & $\begin{array}{c}2.793 \\
\pm 0.135\end{array}$ & $\begin{array}{l}3.99 \\
\text { bcd }\end{array}$ & $\begin{array}{l}127.6 \\
\pm 10.6\end{array}$ & $\begin{array}{l}37.59 \\
\pm 7.11\end{array}$ & $\begin{array}{r}26.63 \\
\pm 5.33\end{array}$ & $\begin{array}{c}63.92 \\
\text { de }\end{array}$ & $\begin{array}{c}92.0 \\
\pm 3.51\end{array}$ & $\begin{array}{c}55.667 \\
\pm 0.88\end{array}$ & $\begin{array}{c}38.228 \\
\pm 0.33\end{array}$ & $\begin{array}{c}62.00 \\
\mathrm{de}\end{array}$ & $\begin{array}{l}3.257 \\
\pm 0.33\end{array}$ & $\begin{array}{l}4.000 \\
\pm 0.01\end{array}$ & $\begin{array}{l}3.333 \\
\pm 0.32\end{array}$ & $\begin{array}{c}3.55 \\
a b\end{array}$ & $\begin{array}{l}1.675 \\
\pm 0.03\end{array}$ & $\begin{array}{l}1.405 \\
\pm 0.03\end{array}$ & $\begin{array}{l}1.301 \\
\pm 0.02\end{array}$ & $\begin{array}{c}1.460 \\
b\end{array}$ \\
\hline BG6 & $\begin{array}{c}5.694 \\
\pm 0.322\end{array}$ & $\begin{array}{c}4.358 \\
\pm 0.181\end{array}$ & $\begin{array}{c}3.127 \\
\pm 0.202\end{array}$ & $\begin{array}{c}4.39 \\
\mathrm{ab}\end{array}$ & $\begin{array}{c}158.00 \\
\pm 32.0\end{array}$ & $\begin{array}{c}107.67 \\
\pm 6.14\end{array}$ & $\begin{array}{l}55.27 \\
\pm 3.10\end{array}$ & $\begin{array}{c}106.97 \\
\mathrm{a}\end{array}$ & $\begin{array}{c}107.00 \\
\pm 4.51\end{array}$ & $\begin{array}{c}59.00 \\
\pm 0.557\end{array}$ & $\begin{array}{l}41.67 \\
\pm 1.67\end{array}$ & $\begin{array}{c}69.22 \\
\text { bc }\end{array}$ & $\begin{array}{l}3.325 \\
\pm 0.33\end{array}$ & $\begin{array}{l}3.313 \\
\pm 0.33\end{array}$ & $\begin{array}{l}3.000 \\
\pm 0.01\end{array}$ & $\begin{array}{l}3.22 \\
\text { bcd }\end{array}$ & $\begin{array}{l}1.787 \\
\pm 0.08\end{array}$ & $\begin{array}{l}1.572 \\
\pm 0.03\end{array}$ & $\begin{array}{r}1.389 \\
\pm 0.06\end{array}$ & $\begin{array}{c}1.583 \\
\mathrm{a}\end{array}$ \\
\hline BG21 & $\begin{array}{l}4.708 \\
\pm 0.43\end{array}$ & $\begin{array}{l}3.633 \\
\pm 0.30\end{array}$ & $\begin{array}{l}2.646 \\
\pm 0.22\end{array}$ & $\begin{array}{c}3.66 \\
d\end{array}$ & $\begin{array}{r}112.4 \\
\pm 16.2\end{array}$ & $\begin{array}{r}35.99 \\
\pm 3.09\end{array}$ & $\begin{array}{c}9.94 \\
\pm 0.36\end{array}$ & $\begin{array}{c}52.78 \\
\text { ef }\end{array}$ & $\begin{array}{l}79.67 \\
\pm 3.53\end{array}$ & $\begin{array}{c}61.333 \\
\pm 0.66\end{array}$ & $\begin{array}{r}45.33 \\
\pm 1.45\end{array}$ & $\begin{array}{c}62.11 \\
\text { de }\end{array}$ & $\begin{array}{l}4.000 \\
\pm 0.01\end{array}$ & $\begin{array}{l}3.667 \\
\pm 0.33\end{array}$ & $\begin{array}{l}3.647 \\
\pm 0.33\end{array}$ & $\begin{array}{c}3.77 \\
\mathrm{a}\end{array}$ & $\begin{array}{l}1.669 \\
\pm 0.07\end{array}$ & $\begin{array}{r}1.435 \\
\pm 0.02\end{array}$ & $\begin{array}{l}1.297 \\
\pm 0.05\end{array}$ & $\begin{array}{c}1.467 \\
\mathrm{~b}\end{array}$ \\
\hline BG7 & $\begin{array}{c}4.848 \\
\pm 0.342\end{array}$ & $\begin{array}{c}3.700 \\
\pm 0.336\end{array}$ & $\begin{array}{c}2.724 \\
\pm 0.164\end{array}$ & $\begin{array}{c}3.75 \\
\mathrm{~cd}\end{array}$ & $\begin{array}{r}134.4 \\
\pm 22.3\end{array}$ & $\begin{array}{c}80.8 \\
\pm 21.3\end{array}$ & $\begin{array}{l}23.36 \\
\pm 2.81\end{array}$ & $\begin{array}{c}79.53 \\
\text { bcd }\end{array}$ & $\begin{array}{c}108.00 \\
\pm 12.1\end{array}$ & $\begin{array}{l}62.67 \\
\pm 1.45\end{array}$ & $\begin{array}{c}41.667 \\
\pm 0.66\end{array}$ & $\begin{array}{c}70.77 \\
\text { b }\end{array}$ & $\begin{array}{l}3.667 \\
\pm 0.66\end{array}$ & $\begin{array}{l}3.667 \\
\pm 0.33\end{array}$ & $\begin{array}{l}3.621 \\
\pm 0.31\end{array}$ & $\begin{array}{c}3.66 \\
a b\end{array}$ & $\begin{array}{l}1.618 \\
\pm 0.02\end{array}$ & $\begin{array}{l}1.165 \\
\pm 0.05\end{array}$ & $\begin{array}{l}1.257 \\
\pm 0.01\end{array}$ & $\begin{array}{c}1.347 \\
\mathrm{c}\end{array}$ \\
\hline BG12 & $\begin{array}{c}4.942 \\
\pm 0.285 \\
\end{array}$ & $\begin{array}{c}3.673 \\
\pm 0.226 \\
\end{array}$ & $\begin{array}{c}2.743 \\
\pm 0.158 \\
\end{array}$ & $\begin{array}{c}3.78 \\
\mathrm{~cd}\end{array}$ & $\begin{array}{r}145.6 \\
\pm 14.1 \\
\end{array}$ & $\begin{array}{c}35.1 \\
\pm 11.1 \\
\end{array}$ & $\begin{array}{r}13.28 \\
\pm 1.57 \\
\end{array}$ & $\begin{array}{c}64.66 \\
\mathrm{de}\end{array}$ & $\begin{array}{c}109.00 \\
\pm 4.93 \\
\end{array}$ & $\begin{array}{r}66.00 \\
\pm 0.57 \\
\end{array}$ & $\begin{array}{c}44.317 \\
\pm 0.66\end{array}$ & $\begin{array}{c}73.11 \\
\text { ab }\end{array}$ & $\begin{array}{l}3.661 \\
\pm 0.33\end{array}$ & $\begin{array}{l}3.526 \\
\pm 0.23\end{array}$ & $\begin{array}{c}3.24 \\
\pm 0.30\end{array}$ & $\begin{array}{c}3.66 \\
\mathrm{ab}\end{array}$ & $\begin{array}{l}1.537 \\
\pm 0.09\end{array}$ & $\begin{array}{r}1.413 \\
\pm 0.03\end{array}$ & $\begin{array}{l}1.194 \\
\pm 0.07\end{array}$ & $\begin{array}{c}1.381 \\
\mathrm{bc}\end{array}$ \\
\hline BG9 & $\begin{array}{c}5.893 \\
\pm 0.307 \\
\end{array}$ & $\begin{array}{c}4.446 \\
\pm 0.120\end{array}$ & $\begin{array}{c}3.271 \\
\pm 0.171 \\
\end{array}$ & $\begin{array}{c}4.53 \\
\mathrm{a} \\
\end{array}$ & $\begin{array}{r}192.1 \\
\pm 25.4\end{array}$ & $\begin{array}{c}81.8 \\
\pm 20.6\end{array}$ & $\begin{array}{r}38.98 \\
\pm 0.56\end{array}$ & $\begin{array}{c}104.28 \\
\mathrm{a}\end{array}$ & $\begin{array}{c}118.17 \\
\pm 7.45 \\
\end{array}$ & $\begin{array}{r}71.67 \\
\pm 1.67 \\
\end{array}$ & $\begin{array}{c}46.324 \\
\pm 0.88\end{array}$ & $\begin{array}{c}78.77 \\
\mathrm{a} \\
\end{array}$ & $\begin{array}{r}3.660 \\
\pm 0.33\end{array}$ & $\begin{array}{l}3.521 \\
\pm 0.21\end{array}$ & $\begin{array}{l}3.224 \\
\pm 0.26\end{array}$ & $\begin{array}{c}3.66 \\
a b\end{array}$ & $\begin{array}{r}1.704 \\
\pm 0.07 \\
\end{array}$ & $\begin{array}{l}1.324 \\
\pm 0.05\end{array}$ & $\begin{array}{r}1.313 \\
\pm 0.12\end{array}$ & $\begin{array}{c}1.447 \\
\text { bc }\end{array}$ \\
\hline BR21 & $\begin{array}{c}5.448 \\
\pm 0.668 \\
\end{array}$ & $\begin{array}{c}3.867 \\
\pm 0.212 \\
\end{array}$ & $\begin{array}{c}3.057 \\
\pm 0.348 \\
\end{array}$ & $\begin{array}{l}4.12 \\
\text { abcd }\end{array}$ & $\begin{array}{r}135.1 \\
\pm 29.4 \\
\end{array}$ & $\begin{array}{r}35.80 \\
\pm 1.53 \\
\end{array}$ & $\begin{array}{r}24.18 \\
\pm 4.38 \\
\end{array}$ & $\begin{array}{c}65.02 \\
\mathrm{de}\end{array}$ & $\begin{array}{r}87.00 \\
\pm 8.33 \\
\end{array}$ & $\begin{array}{r}63.42 \\
\pm 4.06 \\
\end{array}$ & $\begin{array}{r}40.00 \\
\pm 0.57 \\
\end{array}$ & $\begin{array}{c}63.44 \\
\mathrm{~cd}\end{array}$ & $\begin{array}{r}3.000 \\
\pm 0.01 \\
\end{array}$ & $\begin{array}{r}3.333 \\
\pm 0.33 \\
\end{array}$ & $\begin{array}{r}3.667 \\
\pm 0.33 \\
\end{array}$ & $\begin{array}{l}3.33 \\
\text { abc }\end{array}$ & $\begin{array}{r}1.739 \\
\pm 0.05 \\
\end{array}$ & $\begin{array}{l}1.202 \\
\pm 0.04 \\
\end{array}$ & $\begin{array}{l}1.351 \\
\pm 0.04 \\
\end{array}$ & $\begin{array}{c}1.431 \\
\text { bc }\end{array}$ \\
\hline BR16 & $\begin{array}{c}5.736 \\
\pm 0.371 \\
\end{array}$ & $\begin{array}{c}4.457 \\
\pm 0.288 \\
\end{array}$ & $\begin{array}{c}3.517 \\
\pm 0.167 \\
\end{array}$ & $\begin{array}{c}4.57 \\
\mathrm{a} \\
\end{array}$ & $\begin{array}{c}128.29 \\
\pm 4.67 \\
\end{array}$ & $\begin{array}{r}47.06 \\
\pm 6.65 \\
\end{array}$ & $\begin{array}{r}31.09 \\
\pm 5.70 \\
\end{array}$ & $\begin{array}{c}68.81 \\
\text { cde }\end{array}$ & $\begin{array}{r}69.33 \\
\pm 1.20 \\
\end{array}$ & $\begin{array}{r}52.57 \\
\pm 1.20 \\
\end{array}$ & $\begin{array}{c}45.667 \\
\pm 0.88 \\
\end{array}$ & $\begin{array}{c}55.77 \\
\mathrm{f} \\
\end{array}$ & $\begin{array}{r}2.326 \\
\pm 0.23 \\
\end{array}$ & $\begin{array}{l}3.667 \\
\pm 0.35 \\
\end{array}$ & $\begin{array}{r}3.00 \\
\pm 0.33 \\
\end{array}$ & $\begin{array}{c}3.00 \\
\mathrm{~cd}\end{array}$ & $\begin{array}{r}1.693 \\
\pm 0.03 \\
\end{array}$ & $\begin{array}{l}1.315 \\
\pm 0.02 \\
\end{array}$ & $\begin{array}{r}1.207 \\
\pm 0.07 \\
\end{array}$ & $\begin{array}{c}1.405 \\
\text { bc }\end{array}$ \\
\hline BR20 & $\begin{array}{c}5.342 \\
\pm 0.572 \\
\end{array}$ & $\begin{array}{c}4.084 \\
\pm 0.448 \\
\end{array}$ & $\begin{array}{c}3.298 \\
\pm 0.147 \\
\end{array}$ & $\begin{array}{l}4.24 \\
\mathrm{abc}\end{array}$ & $\begin{array}{c}118.30 \\
\pm 8.26\end{array}$ & $\begin{array}{c}108.13 \\
\pm 2.12\end{array}$ & $\begin{array}{r}27.37 \\
\pm 3.06 \\
\end{array}$ & $\begin{array}{c}87.92 \\
\text { abc }\end{array}$ & $\begin{array}{r}64.62 \\
\pm 3.84\end{array}$ & $\begin{array}{r}56.35 \\
\pm 3.38\end{array}$ & $\begin{array}{l}44.75 \\
\pm 0.66\end{array}$ & $\begin{array}{c}55.00 \\
\mathrm{f}\end{array}$ & $\begin{array}{l}3.524 \\
\pm 0.35\end{array}$ & $\begin{array}{l}3.621 \\
\pm 0.23 \\
\end{array}$ & $\begin{array}{l}3.629 \\
\pm 0.21\end{array}$ & $\begin{array}{c}3.55 \\
a b\end{array}$ & $\begin{array}{l}1.651 \\
\pm 0.06\end{array}$ & $\begin{array}{l}1.283 \\
\pm 0.05\end{array}$ & $\begin{array}{l}1.110 \\
\pm 0.01\end{array}$ & $\begin{array}{c}1.348 \\
\mathrm{c}\end{array}$ \\
\hline BR15 & $\begin{array}{c}5.817 \\
\pm 0.388\end{array}$ & $\begin{array}{c}4.221 \\
\pm 0.069\end{array}$ & $\begin{array}{c}3.562 \\
\pm 0.243\end{array}$ & $\begin{array}{c}4.53 \\
\mathrm{a}\end{array}$ & $\begin{array}{r}75.09 \\
\pm 3.98\end{array}$ & $\begin{array}{l}26.15 \\
\pm 3.31\end{array}$ & $\begin{array}{l}15.62 \\
\pm 0.98\end{array}$ & $\begin{array}{c}38.95 \\
\mathrm{f}\end{array}$ & $\begin{array}{l}45.00 \\
\pm 3.06\end{array}$ & $\begin{array}{l}55.67 \\
\pm 1.20\end{array}$ & $\begin{array}{l}42.00 \\
\pm 0.57\end{array}$ & $\begin{array}{c}47.55 \\
\mathrm{q}\end{array}$ & $\begin{array}{c}2.34 \\
\pm 0.33\end{array}$ & $\begin{array}{l}3.654 \\
\pm 0.33\end{array}$ & $\begin{array}{l}3.667 \\
\pm 0.33\end{array}$ & $\begin{array}{l}3.22 \\
\text { bcd }\end{array}$ & $\begin{array}{l}1.719 \\
\pm 0.06\end{array}$ & $\begin{array}{l}1.335 \\
\pm 0.05\end{array}$ & $\begin{array}{c}1.184 \\
\pm 0.04\end{array}$ & $\begin{array}{c}1.413 \\
b c\end{array}$ \\
\hline BR27 & $\begin{array}{c}5.691 \\
\pm 0.547\end{array}$ & $\begin{array}{c}4.089 \\
\pm 0.101\end{array}$ & $\begin{array}{c}3.492 \\
\pm 0.288\end{array}$ & $\begin{array}{c}4.24 \\
\mathrm{ab}\end{array}$ & $\begin{array}{r}147.3 \\
\pm 13.9\end{array}$ & $\begin{array}{c}92.7 \\
\pm 17.8\end{array}$ & $\begin{array}{l}35.86 \\
\pm 4.03\end{array}$ & $\begin{array}{c}91.95 \\
\mathrm{ab}\end{array}$ & $\begin{array}{r}65.55 \\
\pm 2.89\end{array}$ & $\begin{array}{l}51.23 \\
\pm 0.57\end{array}$ & $\begin{array}{l}35.56 \\
\pm 2.00\end{array}$ & $\begin{array}{c}50.33 \\
\text { fg }\end{array}$ & $\begin{array}{l}2.667 \\
\pm 0.33\end{array}$ & $\begin{array}{c}3.00 \\
\pm 0.01\end{array}$ & $\begin{array}{l}2.645 \\
\pm 0.33\end{array}$ & $\begin{array}{c}2.77 \\
\mathrm{~d}\end{array}$ & $\begin{array}{l}1.645 \\
\pm 0.57\end{array}$ & $\begin{array}{c}1.278 \\
\pm 0.44\end{array}$ & $\begin{array}{c}1.203 \\
\pm 0.14\end{array}$ & $\begin{array}{c}1.375 \\
b c\end{array}$ \\
\hline BR4 & $\begin{array}{c}5.569 \\
\pm 0.574 \\
\end{array}$ & $\begin{array}{c}3.994 \\
\pm 0.143 \\
\end{array}$ & $\begin{array}{c}3.424 \\
\pm 0.388 \\
\end{array}$ & $\begin{array}{c}4.32 \\
\mathrm{ab}\end{array}$ & $\begin{array}{c}101.50 \\
\pm 10.6\end{array}$ & $\begin{array}{l}39.60 \\
\pm 10.4\end{array}$ & $\begin{array}{l}23.69 \\
\pm 0.24\end{array}$ & $\begin{array}{c}54.94 \\
\text { ef }\end{array}$ & $\begin{array}{r}72.33 \\
\pm 3.38 \\
\end{array}$ & $\begin{array}{l}56.33 \\
\pm 0.33\end{array}$ & $\begin{array}{l}40.00 \\
\pm 0.57\end{array}$ & $\begin{array}{c}56.22 \\
\text { ef }\end{array}$ & $\begin{array}{l}2.667 \\
\pm 0.33\end{array}$ & $\begin{array}{r}3.662 \\
\pm 0.33\end{array}$ & $\begin{array}{l}3.541 \\
\pm 0.33\end{array}$ & $\begin{array}{l}3.33 \\
a b c\end{array}$ & $\begin{array}{l}1.808 \\
\pm 0.03\end{array}$ & $\begin{array}{l}1.404 \\
\pm 0.02\end{array}$ & $\begin{array}{l}1.166 \\
\pm 0.14\end{array}$ & $\begin{array}{c}1.459 \\
\mathrm{~b}\end{array}$ \\
\hline GM & $5.36 \mathrm{a}$ & $4.01 \mathrm{~b}$ & $3.10 \mathrm{c}$ & & $132.2 \mathrm{a}$ & $58.90 \mathrm{~b}$ & $26.88 \mathrm{c}$ & & $85.35 \mathrm{a}$ & $60.02 \mathrm{~b}$ & $41.97 \mathrm{c}$ & & $3.58 \mathrm{a}$ & $3.46 \mathrm{a}$ & $3.20 \mathrm{~b}$ & & $1.67 \mathrm{a}$ & $1.35 \mathrm{~b}$ & $1.24 \mathrm{c}$ & \\
\hline LSD g & \multicolumn{4}{|c|}{0.488} & \multicolumn{4}{|c|}{19.903} & \multicolumn{4}{|c|}{6.164} & \multicolumn{4}{|c|}{0.529} & \multicolumn{4}{|c|}{0.107} \\
\hline LSD E & \multicolumn{4}{|c|}{0.234} & \multicolumn{4}{|c|}{9.561} & \multicolumn{4}{|c|}{2.961} & \multicolumn{4}{|c|}{0.254} & \multicolumn{4}{|c|}{0.0517} \\
\hline $\begin{array}{l}\mathrm{GM}=\mathrm{g} \\
\mathrm{g}=\text { gen }\end{array}$ & d mean & & & & & & & & & & & & & & & & & & & \\
\hline
\end{tabular}




\begin{tabular}{|c|c|c|c|c|c|c|c|c|c|c|c|c|c|c|c|c|c|c|c|c|}
\hline \multicolumn{5}{|c|}{\begin{tabular}{c|c} 
Cont.I Table (5): Means for various seed \\
\end{tabular}} & \multicolumn{4}{|c|}{ No. of total pods/plant } & \multicolumn{4}{|c|}{ Germination percentage } & \multicolumn{4}{|c|}{ Root length } & \multicolumn{4}{|c|}{ Shoot length } \\
\hline & E1 & E2 & E3 & GM & E1 & E2 & E3 & GM & E1 & E2 & E3 & GM & E1 & E2 & E3 & GM & E1 & E2 & E3 & GM \\
\hline BG4 & $\begin{array}{l}3.533 \\
\pm 0.20\end{array}$ & $\begin{array}{l}2.779 \\
\pm 0.21\end{array}$ & $\begin{array}{l}1.961 \\
\pm 0.11\end{array}$ & $\begin{array}{c}2.75 \\
\text { ef }\end{array}$ & $\begin{array}{l}28.67 \\
\pm 3.18\end{array}$ & $\begin{array}{c}8.73 \\
\pm 1.11\end{array}$ & $\begin{array}{c}7.01 \\
\pm 1.65\end{array}$ & $\begin{array}{c}14.80 \\
\text { c }\end{array}$ & $\begin{array}{l}92.66 \\
\pm 0.66\end{array}$ & $\begin{array}{r}46.00 \\
\pm 2.08\end{array}$ & $\begin{array}{l}31.35 \\
\pm 1.33\end{array}$ & $\begin{array}{c}56.66 \\
\text { bc }\end{array}$ & $\begin{array}{c}9.169 \\
0.59\end{array}$ & $\begin{array}{l}5.867 \\
\pm 0.39\end{array}$ & $\begin{array}{l}3.355 \\
\pm 0.10\end{array}$ & $\begin{array}{c}6.13 \\
a\end{array}$ & $\begin{array}{c}14.106 \\
\pm 0.30\end{array}$ & $\begin{array}{c}11.224 \\
0.19 \pm\end{array}$ & $\begin{array}{l}4.405 \\
0.20 \pm\end{array}$ & $\begin{array}{c}9.91 \\
\text { ab }\end{array}$ \\
\hline BG14 & $\begin{array}{l}3.366 \\
\pm 0.08\end{array}$ & $\begin{array}{l}2.649 \\
\pm 0.04\end{array}$ & $\begin{array}{r}1.868 \\
\pm 0.04\end{array}$ & $\begin{array}{c}2.62 \\
\text { fg }\end{array}$ & $\begin{array}{r}25.00 \\
\pm 1.53\end{array}$ & $\begin{array}{c}9.65 \\
\pm 2.21\end{array}$ & $\begin{array}{c}9.35 \\
\pm 1.64\end{array}$ & $\begin{array}{c}14.66 \\
\text { c }\end{array}$ & $\begin{array}{r}97.67 \\
\pm 1.45\end{array}$ & $\begin{array}{l}58.66 \\
\pm 0.88\end{array}$ & $\begin{array}{r}29.67 \\
\pm 1.20\end{array}$ & $\begin{array}{c}62.0 \\
a\end{array}$ & $\begin{array}{r}5.959 \\
\pm 0.91\end{array}$ & $\begin{array}{l}5.022 \\
\pm 0.39\end{array}$ & $\begin{array}{r}\mathbf{3 . 3 2 7} \\
\mathbf{\pm 0 . 0 9}\end{array}$ & $\begin{array}{c}4.76 \\
\text { bc }\end{array}$ & $\begin{array}{c}14.794 \\
\pm 0.22\end{array}$ & $\begin{array}{c}10.336 \\
\pm 0.42\end{array}$ & $\begin{array}{l}4.720 \\
\pm 0.37\end{array}$ & $\begin{array}{c}9.96 \\
\text { ab }\end{array}$ \\
\hline BG6 & $\begin{array}{l}3.733 \\
\pm 0.18\end{array}$ & $\begin{array}{r}2.867 \\
\pm 0.21\end{array}$ & $\begin{array}{r}2.072 \\
\pm 0.10\end{array}$ & $\begin{array}{c}2.89 \\
\text { de }\end{array}$ & $\begin{array}{l}27.45 \\
\pm 3.93\end{array}$ & $\begin{array}{r}24.40 \\
\pm 1.26\end{array}$ & $\begin{array}{r}17.56 \\
\pm 1.13\end{array}$ & $\begin{array}{c}23.09 \\
\text { a }\end{array}$ & $\begin{array}{r}92.00 \\
\pm 1.15\end{array}$ & $\begin{array}{r}46.57 \\
\pm 1.45\end{array}$ & $\begin{array}{r}27.34 \\
\pm 2.85\end{array}$ & $\begin{array}{c}55.22 \\
\text { c }\end{array}$ & $\begin{array}{r}6.881 \\
\pm 0.31\end{array}$ & $\begin{array}{r}3.676 \\
\pm 0.29\end{array}$ & $\begin{array}{r}2.139 \\
\pm 0.29\end{array}$ & $\begin{array}{r}4.23 \\
\text { def }\end{array}$ & $\begin{array}{c}15.280 \\
\pm 0.15\end{array}$ & $\begin{array}{c}10.523 \\
\pm 0.55\end{array}$ & $\begin{array}{l}4.136 \\
\pm 0.05\end{array}$ & $\begin{array}{c}9.97 \\
\text { ab }\end{array}$ \\
\hline BG21 & $\begin{array}{r}2.966 \\
\pm 0.03\end{array}$ & $\begin{array}{l}2.371 \\
\pm 0.06\end{array}$ & $\begin{array}{r}1.646 \\
\pm 0.02\end{array}$ & $\begin{array}{c}2.32 \\
\text { h }\end{array}$ & $\begin{array}{l}23.67 \\
\pm 1.20\end{array}$ & $\begin{array}{r}9.855 \\
\pm 0.29\end{array}$ & $\begin{array}{l}3.844 \\
\pm 0.21\end{array}$ & $\begin{array}{c}12.45 \\
\text { c }\end{array}$ & $\begin{array}{r}92.53 \\
\pm 0.57\end{array}$ & $\begin{array}{r}45.95 \\
\pm 0.57\end{array}$ & $\begin{array}{l}26.74 \\
\pm 0.57\end{array}$ & $\begin{array}{c}54.33 \\
\text { c }\end{array}$ & $\begin{array}{r}5.933 \\
\pm 0.37\end{array}$ & $\begin{array}{r}5.066 \\
\pm 0.31\end{array}$ & $\begin{array}{r}2.501 \\
\pm 0.09\end{array}$ & $\begin{array}{l}4.50 \\
\text { cde }\end{array}$ & $\begin{array}{c}14.254 \\
\pm 0.57\end{array}$ & $\begin{array}{c}10.654 \\
\pm 0.35\end{array}$ & $\begin{array}{r}4.949 \\
\pm 0.06\end{array}$ & $\begin{array}{c}9.95 \\
\text { ab }\end{array}$ \\
\hline BG7 & $\begin{array}{r}3.200 \\
\pm 0.20\end{array}$ & $\begin{array}{r}2.486 \\
\pm 0.15\end{array}$ & $\begin{array}{r}1.776 \\
\pm 0.11\end{array}$ & $\begin{array}{c}2.48 \\
\mathrm{gh}\end{array}$ & $\begin{array}{r}27.67 \\
\pm 4.26\end{array}$ & $\begin{array}{r}20.88 \\
\pm 4.12\end{array}$ & $\begin{array}{l}8.626 \\
\pm 0.54\end{array}$ & $\begin{array}{c}19.05 \\
\text { b }\end{array}$ & $\begin{array}{r}97.67 \\
\pm 1.45\end{array}$ & $\begin{array}{l}52.42 \\
\pm 1.45\end{array}$ & $\begin{array}{r}23.47 \\
\pm 1.20\end{array}$ & $\begin{array}{c}57.77 \\
\text { b }\end{array}$ & $\begin{array}{r}8.107 \\
\pm 0.39\end{array}$ & $\begin{array}{r}5.274 \\
\pm 0.04\end{array}$ & $\begin{array}{r}2.425 \\
\pm 0.06\end{array}$ & $\begin{array}{c}5.23 \\
\text { b }\end{array}$ & $\begin{array}{c}16.482 \\
\pm 0.59\end{array}$ & $\begin{array}{c}10.055 \\
\pm 0.27\end{array}$ & $\begin{array}{r}4.409 \\
\pm 0.12\end{array}$ & $\begin{array}{c}10.31 \\
\mathrm{a}\end{array}$ \\
\hline BG12 & $\begin{array}{l}3.066 \\
\pm 0.06\end{array}$ & $\begin{array}{r}2.416 \\
\pm 0.04\end{array}$ & $\begin{array}{l}1.702 \\
\pm 0.03\end{array}$ & $\begin{array}{c}2.39 \\
\mathrm{~h}\end{array}$ & $\begin{array}{r}29.84 \\
\pm 1.20\end{array}$ & $\begin{array}{c}8.93 \\
\pm 2.38\end{array}$ & $\begin{array}{l}4.870 \\
\pm 0.60\end{array}$ & $\begin{array}{c}14.37 \\
\text { c }\end{array}$ & $\begin{array}{l}88.67 \\
\pm 1.33\end{array}$ & $\begin{array}{l}60.65 \\
\pm 0.66\end{array}$ & $\begin{array}{l}20.00 \\
\pm 1.15\end{array}$ & $\begin{array}{c}56.44 \\
\text { bc }\end{array}$ & $\begin{array}{l}5.992 \\
\pm 0.38\end{array}$ & $\begin{array}{l}4.634 \\
\pm 0.11\end{array}$ & $\begin{array}{l}1.590 \\
\pm 0.20\end{array}$ & $\begin{array}{c}4.07 \\
\text { ef }\end{array}$ & $\begin{array}{c}15.682 \\
\pm 0.30\end{array}$ & $\begin{array}{l}9.326 \\
\pm 0.33\end{array}$ & $\begin{array}{l}3.022 \\
\pm 0.37\end{array}$ & $\begin{array}{l}9.34 \\
\text { bcd }\end{array}$ \\
\hline BG9 & $\begin{array}{l}3.800 \\
\pm 0.15\end{array}$ & $\begin{array}{l}3.053 \\
\pm 0.19\end{array}$ & $\begin{array}{l}2.109 \\
\pm 0.08\end{array}$ & $\begin{array}{c}2.98 \\
\text { cd }\end{array}$ & $\begin{array}{l}32.26 \\
\pm 2.91\end{array}$ & $\begin{array}{l}17.53 \\
\pm 3.87\end{array}$ & $\begin{array}{c}11.997 \\
\pm 0.76\end{array}$ & $\begin{array}{c}20.62 \\
\text { ab }\end{array}$ & $\begin{array}{r}97.00 \\
\pm 2.52\end{array}$ & $\begin{array}{l}54.51 \\
\pm 0.66\end{array}$ & $\begin{array}{r}16.00 \\
\pm 2.08\end{array}$ & $\begin{array}{c}55.77 \\
\text { bc }\end{array}$ & $\begin{array}{l}6.795 \\
\pm 0.39\end{array}$ & $\begin{array}{l}4.474 \\
\pm 0.11\end{array}$ & $\begin{array}{l}1.219 \\
\pm 0.20\end{array}$ & $\begin{array}{c}4.16 \\
\text { ef }\end{array}$ & $\begin{array}{c}15.252 \\
\pm 0.68\end{array}$ & $\begin{array}{l}9.637 \\
\pm 0.22\end{array}$ & $\begin{array}{l}3.082 \\
\pm 0.30\end{array}$ & $\begin{array}{l}9.32 \\
\text { bcd }\end{array}$ \\
\hline BR21 & $\begin{array}{r}3.733 \\
\pm 0.13 \\
\end{array}$ & $\begin{array}{l}3.001 \\
\pm 0.20 \\
\end{array}$ & $\begin{array}{r}2.072 \\
\pm 0.07 \\
\end{array}$ & $\begin{array}{l}2.95 \\
\text { cde }\end{array}$ & $\begin{array}{r}25.67 \\
\pm 6.49 \\
\end{array}$ & $\begin{array}{l}8.631 \\
\pm 0.76 \\
\end{array}$ & $\begin{array}{c}8.45 \\
\pm 2.36\end{array}$ & $\begin{array}{c}14.24 \\
\text { c }\end{array}$ & $\begin{array}{r}88.33 \\
\pm 2.73 \\
\end{array}$ & $\begin{array}{r}49.45 \\
\pm 0.66 \\
\end{array}$ & $\begin{array}{r}17.32 \\
\pm 1.20\end{array}$ & $\begin{array}{c}51.66 \\
d\end{array}$ & $\begin{array}{r}6.462 \\
\pm 0.40 \\
\end{array}$ & $\begin{array}{r}3.808 \\
\pm 0.21 \\
\end{array}$ & $\begin{array}{r}2.486 \\
\pm 0.08 \\
\end{array}$ & $\begin{array}{l}4.25 \\
\text { def }\end{array}$ & $\begin{array}{c}14.861 \\
\pm 0.23\end{array}$ & $\begin{array}{r}9.396 \\
\pm 0.48\end{array}$ & $\begin{array}{l}3.190 \\
\pm 0.41\end{array}$ & $\begin{array}{c}9.149 \\
\text { cd }\end{array}$ \\
\hline BR16 & $\begin{array}{r}4.033 \\
\pm 0.16 \\
\end{array}$ & $\begin{array}{l}3.201 \\
\pm 0.17 \\
\end{array}$ & $\begin{array}{r}2.238 \\
\pm 0.09 \\
\end{array}$ & $\begin{array}{c}3.15 \\
\text { bc }\end{array}$ & $\begin{array}{r}22.67 \\
\pm 2.33 \\
\end{array}$ & $\begin{array}{r}10.61 \\
\pm 1.48 \\
\end{array}$ & $\begin{array}{c}9.61 \\
\pm 1.22 \\
\end{array}$ & $\begin{array}{c}14.29 \\
\mathrm{c} \\
\end{array}$ & $\begin{array}{r}91.00 \\
\pm 1.00 \\
\end{array}$ & $\begin{array}{l}44.36 \\
\pm 0.88 \\
\end{array}$ & $\begin{array}{r}20.00 \\
\pm 0.57 \\
\end{array}$ & $\begin{array}{c}51.77 \\
\text { d }\end{array}$ & $\begin{array}{r}6.582 \\
\pm 0.33 \\
\end{array}$ & $\begin{array}{r}5.300 \\
\pm 0.19 \\
\end{array}$ & $\begin{array}{l}2.189 \\
\pm 0.27 \\
\end{array}$ & $\begin{array}{c}4.69 \\
\text { cd }\end{array}$ & $\begin{array}{c}15.305 \\
\pm 0.47 \\
\end{array}$ & $\begin{array}{c}10.122 \\
\pm 0.61\end{array}$ & $\begin{array}{r}4.391 \\
\pm 0.29 \\
\end{array}$ & $\begin{array}{c}9.939 \\
\text { ab } \\
\end{array}$ \\
\hline BR20 & $\begin{array}{l}3.933 \\
\pm 0.34\end{array}$ & $\begin{array}{r}3.156 \\
\pm 0.29\end{array}$ & $\begin{array}{r}2.183 \\
\pm 0.19\end{array}$ & $\begin{array}{l}3.09 \\
\text { bed }\end{array}$ & $\begin{array}{r}22.85 \\
\pm 0.88\end{array}$ & $\begin{array}{r}29.06 \\
\pm 2.79\end{array}$ & $\begin{array}{c}9.39 \\
\pm 1.16\end{array}$ & $\begin{array}{c}20.25 \\
\text { ab }\end{array}$ & $\begin{array}{r}92.00 \\
\pm 0.57\end{array}$ & $\begin{array}{l}63.25 \\
\pm 2.40\end{array}$ & $\begin{array}{r}31.62 \\
\pm 1.86\end{array}$ & $\begin{array}{c}62.22 \\
\mathrm{a}\end{array}$ & $\begin{array}{r}7.022 \\
\pm 0.39\end{array}$ & $\begin{array}{l}3.592 \\
\pm 0.02\end{array}$ & $\begin{array}{l}2.002 \\
\pm 0.20\end{array}$ & $\begin{array}{c}4.20 \\
\text { def }\end{array}$ & $\begin{array}{c}15.256 \\
\pm 0.50\end{array}$ & $\begin{array}{l}8.298 \\
\pm 0.21\end{array}$ & $\begin{array}{l}3.056 \\
\pm 0.26\end{array}$ & $\begin{array}{c}8.870 \\
\text { d }\end{array}$ \\
\hline BR15 & $\begin{array}{r}4.200 \\
\pm 0.05\end{array}$ & $\begin{array}{r}3.363 \\
\pm 0.06\end{array}$ & $\begin{array}{l}2.331 \\
\pm 0.03\end{array}$ & $\begin{array}{c}3.29 \\
\text { ab }\end{array}$ & $\begin{array}{r}13.00 \\
\pm 1.00\end{array}$ & $\begin{array}{l}5.781 \\
\pm 0.65\end{array}$ & $\begin{array}{l}4.881 \\
\pm 0.43 \\
\end{array}$ & $\begin{array}{c}7.88 \\
d\end{array}$ & $\begin{array}{r}91.33 \\
\pm 1.67 \\
\end{array}$ & $\begin{array}{r}60.00 \\
\pm 0.57\end{array}$ & $\begin{array}{r}32.00 \\
\pm 1.15\end{array}$ & $\begin{array}{c}61.11 \\
\mathrm{a}\end{array}$ & $\begin{array}{l}\mathbf{5 . 6 9 2} \\
\pm 0.55\end{array}$ & $\begin{array}{l}4.572 \\
\pm 0.01\end{array}$ & $\begin{array}{l}2.644 \\
\pm 0.01\end{array}$ & $\begin{array}{l}4.30 \\
\text { cde }\end{array}$ & $\begin{array}{c}15.029 \\
\pm 0.53\end{array}$ & $\begin{array}{c}10.130 \\
\pm 0.34\end{array}$ & $\begin{array}{r}4.236 \\
\pm 0.16\end{array}$ & $\begin{array}{l}9.79 \\
\text { abc }\end{array}$ \\
\hline BR27 & $\begin{array}{r}4.300 \\
\pm 0.20 \\
\end{array}$ & $\begin{array}{r}3.474 \\
\pm 0.02 \\
\end{array}$ & $\begin{array}{r}2.387 \\
\pm 0.11 \\
\end{array}$ & $\begin{array}{c}3.38 \\
\mathrm{a} \\
\end{array}$ & $\begin{array}{r}26.33 \\
\pm 3.53 \\
\end{array}$ & $\begin{array}{r}21.38 \\
\pm 4.96\end{array}$ & $\begin{array}{c}11.328 \\
\pm 0.33\end{array}$ & $\begin{array}{c}19.67 \\
\text { ab }\end{array}$ & $\begin{array}{r}92.67 \\
\pm 1.20 \\
\end{array}$ & $\begin{array}{r}64.25 \\
\pm 1.33 \\
\end{array}$ & $\begin{array}{r}32.67 \\
\pm 4.06 \\
\end{array}$ & $\begin{array}{c}63.22 \\
\mathrm{a} \\
\end{array}$ & $\begin{array}{r}6.053 \\
\pm 0.26 \\
\end{array}$ & $\begin{array}{c}3.670 \\
0.30\end{array}$ & $\begin{array}{r}2.445 \\
\pm 0.48 \\
\end{array}$ & $\begin{array}{c}4.05 \\
\text { ef }\end{array}$ & $\begin{array}{c}14.520 \\
\pm 0.92\end{array}$ & $\begin{array}{r}9.217 \\
\pm 0.71 \\
\end{array}$ & $\begin{array}{r}3.277 \\
\pm \mathbf{0 . 3 1} \\
\end{array}$ & $\begin{array}{c}9.00 \\
\text { d }\end{array}$ \\
\hline BR4 & $\begin{array}{l}4.400 \\
\pm 0.20\end{array}$ & $\begin{array}{l}3.518 \\
\pm 0.05\end{array}$ & $\begin{array}{l}2.442 \\
\pm 0.11\end{array}$ & $\begin{array}{c}3.45 \\
\text { a }\end{array}$ & $\begin{array}{r}18.33 \\
\pm 1.45\end{array}$ & $\begin{array}{c}9.41 \\
\pm 2.76\end{array}$ & $\begin{array}{l}7.806 \\
\pm 0.68\end{array}$ & $\begin{array}{c}11.85 \\
\text { c }\end{array}$ & $\begin{array}{r}92.67 \\
\pm 1.33\end{array}$ & $\begin{array}{l}63.41 \\
\pm 1.86\end{array}$ & $\begin{array}{r}29.42 \\
\pm 2.33\end{array}$ & $\begin{array}{c}61.77 \\
\mathbf{a}\end{array}$ & $\begin{array}{r}6.168 \\
\pm 0.29\end{array}$ & $\begin{array}{l}3.549 \\
\pm 0.01\end{array}$ & $\begin{array}{l}1.643 \\
\pm 0.22\end{array}$ & $\begin{array}{c}3.78 \\
\text { f }\end{array}$ & $\begin{array}{c}15.131 \\
\pm 0.22\end{array}$ & $\begin{array}{l}8.697 \\
\pm 0.64\end{array}$ & $\begin{array}{l}3.172 \\
\pm 0.08\end{array}$ & $\begin{array}{c}9.00 \\
d\end{array}$ \\
\hline GM & $3.71 \mathrm{a}$ & $2.94 \mathrm{~b}$ & $2.06 \mathrm{c}$ & & 24.79 a & $14.21 \mathrm{~b}$ & $8.82 \mathrm{c}$ & & $92.74 \mathrm{a}$ & $54.46 \mathrm{~b}$ & $25.87 \mathrm{c}$ & & $4.66 \mathrm{a}$ & $4.44 \mathrm{ab}$ & $4.36 \mathrm{~b}$ & & 15.07 a & $9.81 \mathrm{~b}$ & $3.84 \mathrm{c}$ & \\
\hline LSD g & \multicolumn{4}{|c|}{0.228} & \multicolumn{4}{|c|}{3.95} & \multicolumn{4}{|c|}{2.540} & \multicolumn{4}{|c|}{0.512} & \multicolumn{4}{|c|}{0.658} \\
\hline LSD E & \multicolumn{4}{|c|}{0.109} & & 1.89 & & & \multicolumn{4}{|c|}{1.220} & \multicolumn{4}{|c|}{0.246} & \multicolumn{4}{|c|}{0.316} \\
\hline $\begin{array}{l}\text { GM = g } \\
\mathrm{g}=\text { gen } \\
\mathrm{e}=\text { envi }\end{array}$ & $\mathrm{d} m$ & & & & & & & & & & & & & & & & & & & \\
\hline
\end{tabular}


Cont. II Table (5): Means for various seedling and maturity traits of the 13 genotypes at three locations during 2009 - 2010.

\begin{tabular}{|c|c|c|c|c|c|c|c|c|c|c|c|c|}
\hline & \multicolumn{4}{|c|}{$\mathrm{Na}^{+}$ion concentration } & \multicolumn{4}{|c|}{$\mathbf{K}^{+}$ion concentration } & \multicolumn{4}{|c|}{$\mathbf{N a}^{+} / \mathbf{K}^{+}$ion ratio } \\
\hline & E1 & E2 & E3 & GM & E1 & E2 & $\mathbf{E 3}$ & GM & E1 & E2 & $\mathbf{E 3}$ & GM \\
\hline BG4 & $\begin{array}{c}10.204 \\
\pm 0.27\end{array}$ & $\begin{array}{c}12.512 \\
\pm 0.09\end{array}$ & $\begin{array}{c}15.744 \\
\pm 0.29\end{array}$ & $\begin{array}{c}12.82 \\
\text { a }\end{array}$ & $\begin{array}{c}42.172 \\
\pm 0.46\end{array}$ & $\begin{array}{c}30.122 \\
\pm 0.44\end{array}$ & $\begin{array}{c}20.064 \\
\pm 0.19\end{array}$ & $30.78 \mathrm{~g}$ & $\begin{array}{c}0.242 \\
\pm 0.007\end{array}$ & $\begin{array}{c}\mathbf{0 . 4 1 5} \\
\pm \mathbf{0 . 0 0 7}\end{array}$ & $\begin{array}{c}0.784 \\
\pm 0.013\end{array}$ & $0.48 \mathrm{c}$ \\
\hline BG14 & $\begin{array}{c}10.255 \\
\pm 0.05\end{array}$ & $\begin{array}{c}13.259 \\
\pm 0.17\end{array}$ & $\begin{array}{c}15.399 \\
\pm 0.11\end{array}$ & $\begin{array}{c}12.97 \\
\text { a }\end{array}$ & $\begin{array}{c}44.730 \\
\pm 0.29\end{array}$ & $\begin{array}{c}32.557 \\
\pm 0.05\end{array}$ & $\begin{array}{c}20.974 \\
\pm 0.42\end{array}$ & $32.75 \mathrm{~cd}$ & $\begin{array}{l}0.229 \\
\pm 0.01\end{array}$ & $\begin{array}{l}\mathbf{0 . 4 0 7} \\
\pm \mathbf{0 . 0 1}\end{array}$ & $\begin{array}{l}\mathbf{0 . 7 3 4} \\
\pm 0.01\end{array}$ & $0.45 \mathrm{~d}$ \\
\hline BG6 & $\begin{array}{r}11.498 \\
\pm 0.06 \\
\end{array}$ & $\begin{array}{c}12.534 \\
\pm 0.02 \\
\end{array}$ & $\begin{array}{r}14.336 \\
\pm 0.09 \\
\end{array}$ & $\begin{array}{c}12.78 \\
\mathrm{a}\end{array}$ & $\begin{array}{r}42.473 \\
\pm 0.05 \\
\end{array}$ & $\begin{array}{r}35.086 \\
\pm 0.30 \\
\end{array}$ & $\begin{array}{c}18.326 \\
\pm 0.07 \\
\end{array}$ & 31.96 ef & $\begin{array}{r}0.270 \\
\pm 0.01 \\
\end{array}$ & $\begin{array}{r}\mathbf{0 . 3 5 7} \\
\pm \mathbf{0 . 0 2} \\
\end{array}$ & $\begin{array}{r}0.782 \\
\pm 0.01 \\
\end{array}$ & $0.47 \mathrm{~cd}$ \\
\hline BG21 & $\begin{array}{l}7.811 \\
\pm 0.12 \\
\end{array}$ & $\begin{array}{c}10.472 \\
\pm 0.11 \\
\end{array}$ & $\begin{array}{c}14.481 \\
\pm 0.23 \\
\end{array}$ & $\begin{array}{c}10.92 \\
\text { d }\end{array}$ & $\begin{array}{c}\mathbf{4 8 . 8 3 5} \\
\pm 0.77 \\
\end{array}$ & $\begin{array}{c}36.289 \\
\pm 0.29 \\
\end{array}$ & $\begin{array}{c}10.233 \\
\pm 0.45 \\
\end{array}$ & $31.78 \mathrm{f}$ & $\begin{array}{r}0.159 \\
\pm 0.01 \\
\end{array}$ & $\begin{array}{r}0.288 \\
\pm 0.01 \\
\end{array}$ & $\begin{array}{r}1.420 \\
\pm 0.06 \\
\end{array}$ & $0.62 \mathrm{a}$ \\
\hline BG7 & $\begin{array}{l}8.491 \\
\pm 0.06 \\
\end{array}$ & $\begin{array}{c}10.380 \\
\pm 0.09 \\
\end{array}$ & $\begin{array}{r}15.513 \\
\pm 0.13 \\
\end{array}$ & $\begin{array}{c}11.46 \\
\text { c }\end{array}$ & $\begin{array}{c}44.529 \\
\pm 0.21 \\
\end{array}$ & $\begin{array}{r}32.051 \\
\pm 0.26 \\
\end{array}$ & $\begin{array}{c}15.457 \\
\pm 0.12 \\
\end{array}$ & $30.67 \mathrm{~g}$ & $\begin{array}{c}0.190 \\
\pm 0.01 \\
\end{array}$ & $\begin{array}{r}0.323 \\
\pm 0.01 \\
\end{array}$ & $\begin{array}{r}1.003 \\
\pm 0.01 \\
\end{array}$ & $0.50 \mathrm{~b}$ \\
\hline BG12 & $\begin{array}{c}10.563 \\
\pm 0.01\end{array}$ & $\begin{array}{r}12.496 \\
\pm 0.08\end{array}$ & $\begin{array}{r}13.601 \\
\pm 0.18 \\
\end{array}$ & $\begin{array}{c}12.21 \\
\text { b }\end{array}$ & $\begin{array}{r}44.970 \\
\pm 0.32 \\
\end{array}$ & $\begin{array}{c}34.432 \\
\pm 0.43 \\
\end{array}$ & $\begin{array}{c}20.234 \\
\pm 0.28 \\
\end{array}$ & $33.21 \mathrm{c}$ & $\begin{array}{r}0.234 \\
\pm 0.01 \\
\end{array}$ & $\begin{array}{r}\mathbf{0 . 3 6 3} \\
\pm 0.01 \\
\end{array}$ & $\begin{array}{r}0.672 \\
\pm 0.01 \\
\end{array}$ & $0.42 \mathrm{e}$ \\
\hline BG9 & $\begin{array}{l}7.711 \\
\pm 0.07\end{array}$ & $\begin{array}{l}9.689 \\
\pm 0.09\end{array}$ & $\begin{array}{r}12.623 \\
\pm 0.02 \\
\end{array}$ & $\begin{array}{c}10.00 \\
\text { e }\end{array}$ & $\begin{array}{c}39.842 \\
\pm 0.20 \\
\end{array}$ & $\begin{array}{c}35.622 \\
\pm 0.20 \\
\end{array}$ & $\begin{array}{c}20.104 \\
\pm 0.22 \\
\end{array}$ & $31.85 \mathrm{f}$ & $\begin{array}{r}0.193 \\
\pm 0.01 \\
\end{array}$ & $\begin{array}{r}0.272 \\
\pm 0.01 \\
\end{array}$ & $\begin{array}{r}\mathbf{0 . 6 2 8} \\
\pm 0.01 \\
\end{array}$ & $0.36 \mathrm{f}$ \\
\hline BR21 & $\begin{array}{r}9.448 \\
\pm 0.16 \\
\end{array}$ & $\begin{array}{c}10.488 \\
\pm 0.07\end{array}$ & $\begin{array}{c}13.671 \\
\pm 0.14 \\
\end{array}$ & $\begin{array}{c}11.20 \\
\text { cd }\end{array}$ & $\begin{array}{c}44.488 \\
\pm 0.31 \\
\end{array}$ & $\begin{array}{c}32.535 \\
\pm 0.21 \\
\end{array}$ & $\begin{array}{c}18.405 \\
\pm 0.10\end{array}$ & $31.80 \mathrm{f}$ & $\begin{array}{c}0.212 \\
\pm 0.02 \\
\end{array}$ & $\begin{array}{r}0.322 \\
\pm 0.01 \\
\end{array}$ & $\begin{array}{r}0.742 \\
\pm 0.01 \\
\end{array}$ & $0.42 \mathrm{e}$ \\
\hline BR16 & $\begin{array}{c}10.026 \\
\pm 0.28 \\
\end{array}$ & $\begin{array}{c}11.722 \\
\pm 0.28 \\
\end{array}$ & $\begin{array}{c}14.642 \\
\pm 0.03 \\
\end{array}$ & $\begin{array}{c}12.12 \\
\text { b }\end{array}$ & $\begin{array}{c}42.906 \\
\pm 0.40 \\
\end{array}$ & $\begin{array}{r}33.531 \\
\pm 0.05 \\
\end{array}$ & $\begin{array}{r}17.937 \\
\pm \mathbf{0 . 3 5} \\
\end{array}$ & $31.45 \mathrm{f}$ & $\begin{array}{r}\mathbf{0 . 2 3 3} \\
\pm 0.01 \\
\end{array}$ & $\begin{array}{r}0.349 \\
\pm 0.01 \\
\end{array}$ & $\begin{array}{r}0.816 \\
\pm 0.01 \\
\end{array}$ & $0.46 \mathrm{~cd}$ \\
\hline BR20 & $\begin{array}{c}7.51 \\
\pm 1.02 \\
\end{array}$ & $\begin{array}{c}9.528 \\
\pm 0.22 \\
\end{array}$ & $\begin{array}{r}12.562 \\
\pm 0.04 \\
\end{array}$ & $9.86 \mathrm{e}$ & $\begin{array}{l}44.371 \\
\pm 0.25 \\
\end{array}$ & $\begin{array}{c}34.663 \\
\pm 0.10 \\
\end{array}$ & $\begin{array}{c}20.683 \\
\pm 0.15 \\
\end{array}$ & $33.22 \mathrm{c}$ & $\begin{array}{r}\mathbf{0 . 1 6 9} \\
\pm 0.02 \\
\end{array}$ & $\begin{array}{l}0.274 \\
\pm 0.01 \\
\end{array}$ & $\begin{array}{r}\mathbf{0 . 6 0 8} \\
\pm 0.01 \\
\end{array}$ & $0.35 \mathrm{f}$ \\
\hline BR15 & $\begin{array}{r}7.613 \\
\pm 0.95 \\
\end{array}$ & $\begin{array}{c}10.447 \\
\pm 0.09 \\
\end{array}$ & $\begin{array}{c}15.488 \\
\pm 0.28 \\
\end{array}$ & $\begin{array}{c}11.18 \\
\text { cd }\end{array}$ & $\begin{array}{r}45.436 \\
\pm 0.12 \\
\end{array}$ & $\begin{array}{r}32.650 \\
\pm 0.04 \\
\end{array}$ & $\begin{array}{c}19.287 \\
\pm 0.35 \\
\end{array}$ & 32.45 de & $\begin{array}{r}\mathbf{0 . 1 6 7} \\
\pm 0.02 \\
\end{array}$ & $\begin{array}{r}0.319 \\
\pm 0.01 \\
\end{array}$ & $\begin{array}{r}\mathbf{0 . 8 0 4} \\
\pm 0.02 \\
\end{array}$ & $0.43 \mathrm{e}$ \\
\hline BR27 & $\begin{array}{r}9.564 \\
\pm 0.18 \\
\end{array}$ & $\begin{array}{c}11.284 \\
\pm 0.03 \\
\end{array}$ & $\begin{array}{r}15.741 \\
\pm 0.06 \\
\end{array}$ & $\begin{array}{c}12.19 \\
\text { b }\end{array}$ & $\begin{array}{r}\mathbf{4 8 . 5 2} \\
\pm 1.16 \\
\end{array}$ & $\begin{array}{c}35.711 \\
\pm 0.08 \\
\end{array}$ & $\begin{array}{r}29.030 \\
\pm 0.98 \\
\end{array}$ & 37.69 a & $\begin{array}{c}.198 \\
\pm 0.01 \\
\end{array}$ & $\begin{array}{r}0.315 \\
\pm 0.01 \\
\end{array}$ & $\begin{array}{r}\mathbf{0 . 5 4 3} \\
\pm 0.02 \\
\end{array}$ & $0.35 \mathrm{f}$ \\
\hline BR4 & $\begin{array}{c}10.604 \\
\pm 0.03 \\
\end{array}$ & $\begin{array}{r}12.536 \\
\pm 0.16 \\
\end{array}$ & $\begin{array}{c}14.918 \\
\pm 0.02 \\
\end{array}$ & $\begin{array}{c}12.68 \\
\mathrm{a}\end{array}$ & $\begin{array}{c}45.871 \\
\pm 0.28 \\
\end{array}$ & $\begin{array}{r}32.338 \\
\pm 0.38 \\
\end{array}$ & $\begin{array}{r}23.305 \\
\pm 0.26 \\
\end{array}$ & $33.83 \mathrm{~b}$ & $\begin{array}{c}0.231 \\
\pm 0.01 \\
\end{array}$ & $\begin{array}{r}\mathbf{0 . 3 8 7} \\
\pm 0.01 \\
\end{array}$ & $\begin{array}{r}0.640 \\
\pm 0.01 \\
\end{array}$ & $0.41 \mathrm{e}$ \\
\hline GM & $9.33 \mathrm{a}$ & $\begin{array}{c}11.33 \\
\text { b }\end{array}$ & $\begin{array}{c}14.51 \\
\mathrm{a}\end{array}$ & & $\begin{array}{c}44.53 \\
\mathrm{a} \\
\end{array}$ & $33.66 \mathrm{~b}$ & $19.53 \mathrm{c}$ & & $0.21 \mathrm{c}$ & $0.33 \mathrm{~b}$ & $0.78 \mathrm{a}$ & \\
\hline LSD g & & \multicolumn{4}{|c|}{0.580} & \multicolumn{4}{|c|}{0.023} \\
\hline LSD E & & \multicolumn{4}{|c|}{0.278} & \\
\hline
\end{tabular}

GM = grand mean
g= genotypes e = environments E1= Kaha location., E2= Ras Sudr location 1. and E3= Ras Sudr location 2.

Any means within rows or columns followed by the same letter are not statistically different at 0.01 level (Duncan's multiple test)

The remaining 10 genotypes scattered away from the origin in the biplot indicating that the genotypes were more sensitive to environmental interactive forces. Interaction of genotypes with specific environmental conditions was judged by projection of genotype points on to environment spokes. On this basis, the genotypes BG 12 (no. 6), BG 4 (no. 1) and GR 21 (no. 8) had moderate positive interaction and BR 4 (no. 13) and BR 20 (no. 10) had moderate negative interaction under Kaha condition $\left(\mathrm{E}_{1}\right)$. Genotypes BR 20 (no. 10), GR 27 (no. 12), BG 7 (no. 5) and BG 6 (no. 3) had positive interaction and BG 21 (no.4) BR 16 (no. 9) had high negative interaction under Ras Sudr 1 $\left(\mathrm{E}_{2}\right)$ condition. Genotypes BR 15 (no. 11) and BR 4 (no. 13) had high positive interaction and BG 6 (no. 3) had high negative interaction under Ras Sudr $2\left(\mathrm{E}_{3}\right)$ condition. It can be concluded that:

1. The analysis of variance of 13 local okra genotypes in three environments shows that genotype (G), environment (E) and their interaction were significant $(\mathrm{P}<0.01)$ for genotypes. The AMMI model was very effective for studying GEI interaction. The first bilinear AMMI model terms accounted for $71.268 \%$.

2 . No genotype has superior performance in all environments. The biplot shows that the genotypes BG 9 (no. 7), BG6 (no. 3), BR 27 (no. 12) and BR 20 (no. 10) are best-suited for cultivation in a wide range of environments; while, the genotype BR 20 (no. 10) is well suited for cultivation in poor environments.

\subsection{Variation for salinity tolerance in Okra.}

The results for combined analysis of local okra genotypes characteristics across locations is given in Table (5). The salinity conditions (Ras Sudr 1 and Ras Sudr 2 locations) influenced the characteristics of all the genotypes grown under salinity conditions (Ras surd 1 and Ras surd 2) represent the significantly decrease as compared 
to the Kaha condition (The lowest salinity). Genotypes BG 9 and BG 6 in general performed better than the other genotypes across all the three locations / environments. Mean pod weight, yield per plant, plant height, no. of branches/ plant and no. of total pods / plant over locations $(4.53 \mathrm{~g}$, $104.28 \mathrm{~g}, 78.77 \mathrm{~cm}, 3.66$ and $20.62 \mathrm{~g}$, respectively) identified BG 9 as the best yielding genotype and the same genotype (BG 9) recorded the lowest values in $\mathrm{Na}^{+}$ion concentration, $\mathrm{K}^{+}$ion concentration and $\mathrm{Na}^{+} \mathrm{k}^{+}$ion ratio. $(10.00 \mathrm{mM} / \mathrm{L}$, $31.85 \mathrm{~g} /$ plant) as poorest yielding genotype. The highest grand mean of pod weight, yield per plant, plant height, no. of branches / plant, pod diameter, pod length, no. of total pods/plant, germination percentage, root length and shoot length were recorded at Kaha conditions (5.36 g, 132.2 glplant, $85.35 \mathrm{~cm}, 3.58,1.67 \mathrm{~cm}, 3.71 \mathrm{~cm}, 24.79$, $92.74,4.66 \mathrm{~cm}$ and $15.07 \mathrm{~cm}$, repectively). So, this site was conductive / favorable for higher yield. Lowest values for the same characteristics were obtained at Ras Sudr 2 conditions $(3.10 \mathrm{~g}$, 26.88 g plant, $41.97 \mathrm{~cm}, 3.20,1.24 \mathrm{~cm}, 2.06 \mathrm{~cm}$, $8.82,25.87,4.36 \mathrm{~cm}$ and $3.84 \mathrm{~cm}$, respectively) depicting that this site was less conductive /unfavorable for higher yield. Similar results were obtained by Allakhverdiev et al. (2000) who reported that a biotic stresses like heat, cold, drought and salinity effect the plant growth and productivity but the salt stress exerts more drastic effects in terms of low productivity (Munns, 2002). The plant exhibited the lowest germination percentage under salinity condition (Kafi and Goldani, 2001; Jamil and Rho, 2004). The depressed growth of plants may be due to the toxic effect of $\mathrm{Na}^{+}$and $\mathrm{cl}^{-}$ions present in $\mathrm{Nacl}$ and low water potential in the rooting medium (Silveira et al., 2009). It is reported that salt stress effects the plant growth and development by influencing fresh and dry weights of roots, shoot along with shoot length (Ashraf et al., 2003). Growth attributes like plant height, shoot elongation, shoot and root length were severely decreased with salinity. It was noted that plants growing under saline condition remained stunted. The lower water potential in saline soil in turn lower cell tugor causing reduction in cell elongation and cell division (Greenway and Munns, 1980). Although plant height is genetically controlled, environmental factors also have strong influence in the expression of genes (Shahid et al., 2011). The selective uptake of $\mathrm{K}^{+}$in contrast to $\mathrm{Na}^{+}$was considered one of the important physiological mechanisms contributing to salt tolerance in many plant species (Poustini and Siosemardeh, 2004). Okra grown under salinity accumulated maximum amounts of $\mathrm{Na}^{+}$in their leaves and root, so, the growth of these plants was affected due to high concentration of $\mathrm{Na}^{+}$and low ratios of $\mathrm{K}^{+}$(Ahmadi et al., 2009 and Dashti et al., 2009). There was a decrease in $\mathrm{K}^{+}$ concentration both in leaves and roots with increased Nacl salinity in the okra (Shahid et al., 2011). Maintenance of higher $\mathrm{K}^{+} / \mathrm{Na}^{+}$ratio under low salt stress may be one of the reasons for superior growth (Ashraf and Ahmed, 2000). High levels of $\mathrm{K}^{+}$in young leaves are associated with salt tolerance in many plant species (Storey et al., 1993 and Khatum and Flowers, 1995).

It can be concluded that salt stress has affected the Okra plant growth and development $\mathrm{Na}^{+}$reduced the absorption of $\mathrm{K}^{+}$.

From the present investigation, it may be concluded that:

1- The results satisfied one of the breeder's goals for selecting the best -suited genotype for cultivation in a wide salinity range of environments (Kaha, Ras Sudr 1 and Ras Sudr 2).

2- The analysis of variance of 13 local okra genotypes in three environments showed that genotype $(\mathrm{G})$, environment (E) and their interaction were significant $(\mathrm{P}<0.01)$ for genotype.

3- The AMMI model was very effective in studying GEI interaction, the first bilinear AMMI model terms accounted for $71.268 \%$.

4- No genotype had superior performance in all environments.

5- The biplot showed that the genotypes BG 9 (no. 7), BG 6 (no. 3), BR 27 (no. 12) and BR 20 (no. 10) are best-suited for cultivation in a wide range of environments; while, the genotype BR 20 (no. 10) is well suited for cultivation in poor environments.

6- The salt stress affected the plant growth and development. In addition, $\mathrm{Na}^{+}$reduced the absorption of $\mathrm{K}^{+}$.

\section{REFERENCES}

Ahmadi A., Eman Y. and Pessarakli M. (2009). Response of various cultivars of wheat and maize to salinity stress. Journal of food, Agriculture and Environment. 7: 123 - 128.

Allakhverdiev S. I., Sakamoto A., Nishijama Y., Inabe M. and Murata N. (2000). Ionic and osmotic effects of $\mathrm{Na} \mathrm{Cl}$ - induced inactivation of photosystems I and II in Synechococcus sp. Plant Physiology. 123: 1047-1056. 
Annicchiarico P. (2002). Genotype x environment interaction - challenges and opportunities for plant breeding and cultivar recommendations. FAO, Rome, 150pp.

Ashraf M. (1994). Breeding for salinity tolerance in plants. Crit. Rev. Plant Sci. 13: 17-42.

Ashraf M. and Ahmed S. (2000). Influence of sodium chloride on ion accumulation, yield components and fiber characteristics in salt tolerant and salt - sensitive lines of cotton (Gossypium hirsutum L.). Field Crops Research 66: 115 - 127.

Ashraf M., Arfan M. and Ahmed A. (2003). Salt tolerance study in okra: ion relations and gas exchange characteristics. Journal of Plant Nutrition 26: 63 -79.

Becker H.C. and Leon J. (1988). Stability analysis in plant breeding.Plant Breeding, 101: 1-23.

Carbonell S. A., Filho J. A., Dias L. A. Carica A. A. and Morais L.K. (2004). Common bean cultivars and lines interactions with environments. Sci Agric. (Piracicaba, Braz.), 61 (2): 169 - 177.

Chapman H. D. and Pratt P. F. (1961). Methods of analysis for soil, plant and water. University of California, Division of Agriculture Science. P 178, 182.

Dashti A. , Khan A. and Collins J. C. (2009). Effect of salinity on growth, ionic relations and solute content of Sorghum bicolor (M.). Journal of Plant Nutrition. 58: 839 - 843.

Das S., Misra R.C. and Patnaik M.C. (2009). G x $\mathrm{E}$ interaction of mid - late rice genotypes in LR and AMMI model and evaluation of adaptability and yield stability. Environment and Ecology., 27: 529-535.

Eberhart S. A. and Russell W. A., (1966). Stability parameters for comparing varieties. Crop Sci., 6: 36 - 40.

Gabriel K. R. (1971). The biplot graphic display of matrices with application to principal component analysis. Biometrika. 58: 453467.

Gauch H.G. (1988). Model selection and validation for yield trials with interaction. Biometrics. 34: $705-715$.

Gauch H. G. and Zobel R.W. (1988). Predictive and postdictive success of statistical analyses of yield trial. Theor. Appl. Genet 76: $1-10$.

Gauch H. G. and Zobel R.W. (1997). Identifying mega-environments and targeting genotypes. Crop Science 37: $311-326$.

Greenway H. and Munns R. (1980). Mechanism of salt tolerance in non halophytes. Annual
Review of Plant Physiology. 31: 149 -190.

Hamed H. H., Okasha KH. A., Ragab M. E. and Mohamed H. A. (2003). Response of some Okra genotypes (Abelmoschus esculentus L.) to multivariate selection. Arab Univ. J. Sci. 11 (1): $329-342$.

Hariprasanna K., Lab C. and Radhakrishnan T. (2008). G x E interaction and stability analysis in large seeded genotypes of groundnut. J. Oilseeds Res., 25: 126-131.

IRRI (2003). IRRISTAT for windows version 4.4 IRRI, Metro Manila, Philippines.

Jamil M. and Rho E. S. (2004). The effect of salinity (Nacl) on the germination and seedling of sugar beet (Beta vulgaris L.) and cabbage (Brassica oleracea L.) Korean Journal of Plant Research. 7: 226 -232.

Kafi M. and Goldani M., (2001). Effect of water potential and type of osmoticum on seed germination of three crop species of wheat, sugar beet and okra. Agriculture Science and Technology 15: $121-123$.

Khatum S. and Flowers T. J. (1995). Effect of salinity on seed set in rice plant. Cell Environment 18: 61 -87.

Lin C.S., Binns M.R. and lefkovitch L.P. (1986). Stability analysis: where do we stand ? Crop Science. 26: 894-900.

Mahalingam L., Mahendran S., Chanrababu R. and Atlin G. (2006). AMMI analysis for stability of grain yield in rice. Int. J. Bot., 2: 104-106.

Misra R. C., Das S. and Patnaik M. C. (2009). aMMI model analysis of stability and Adaptability of late duration finger millet Eleusine corcana) genotypes. World Applied Science Journal. 6 (12): 1650 1654.

Mohamadi R., Mohammad A., Shabani A. and Daryaei A. (2007). Identification of stability and adaptability in advanced durum genotypes using AMMI analysis. Asian J. Plant Sci., 6: 1261 - 1268.

Munns R. (2002). Comparative physiology of salt and water stress. Plant Cell Environment. 25: 239-250.

Munns R. and James R.A. (2003). Screening method for salinity tolerance: a case study with tetraploid wheat. Plant and Soil. 253: 201-208.

Naveed M., Nadeem M. and Islam N. (2007). AMMI analysis of some upland cotton genotypes for yield stability in different milicus. World I Agril. Sci., 3: 39 -44.

Poustini K. and Siosemardeh A. (2004). Ion 
distribution in wheat cultivars in response to salinity stress. Field Crops Research 85: $125-133$.

Purchase J. L. (1997). Parametric analysis to describe genotype $\mathrm{x}$ environment interaction and yield stability in winter wheat. S. Afri. J. Plant Soil, 17: 101-107.

Shahid M. A., Pervez M. A., Balal R.M. and Ahmed R. (2011). Salt stress effects on some morphological and physiological characteristics of okra (Abelmoschus esculentus L.). Soil Environ. 30 (1): 66-73.

Shinde G. C., Bhingarde M. T.,Khairnkar M.N. and Mahetre S.S. (2002). AMMI analysis for stability of grain yield of pearl millet hybrids. Indian J. Genet., 62: 215 -217.

Siddiq M. A. (1968). Genetics of resistance to cotton leaf curl in sakel cotton. J. Agric. Sci., Comb., 70: 99-103.

Silveira J. A. G., Araujo S. A. M., Lima J. P. M. S. and Viegas A.R. (2009). Roots and leaves display contrasting osmotic adjustment mechanisms in response to $\mathrm{Na}$ cl-salinity in Atriplex mummularia.
Experimental and Environmental Botany. 66: $1-8$.

Storey R. ,Gorham J., Pitman M. C., Hanson M. G. and Gage D. (1993). Response of Melanthera biflora to salinity and water stress. Journal of Experimental Botany 44: $1551-1561$

Tarakanovas P. and Ruzgas V. (2006). Additive main effect and multiplicative interaction analysis of grain yield of wheat varities in Lithuania. Agronmy Research. 4 (1): 91-98.

Vijaykumar C. H. M., Ahmed M. I., viraktamath B. C., Balkrishman R. and Ramesh M. S. (2001). Genotypic x environment effects on yields of rice hybrids in India. Indian $\mathrm{J}$. Genet., 61: $101-106$.

Yan W. and Hunt L. A. (2001). Interpretation of genotype $\mathrm{x}$ environment interaction for winter wheat yield in Ontario. Crop Science. 41: $19-42$.

Zobel R.W., Wright M. G. and Gauch H. G. (1988). Statistical analysis of yield trial. Agron. J. 80: 388 - 393. 
إنتخاب تراكيب وراثيه من الباميه المحليه للثبات تحت الظروف الملحيه

حامد حسن حامد - ححم رائف حافظم

معهد بحوث البساتين ـ مركز البحوث الزر اعيه ـ الجيزه ، * قسم الإنتاج النباتي ـ مركز بحوث الصحر اء ـ القاهره

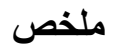

تم زر اعة ثنلاثه عثر تركيبا ور اثيا من أصناف الباميه المحليه ( الأحمر بنوارهو الأخضر البلدي) وهي:

BG4,BG14,BG6,BG21,BG7,BG12,BG9,BR21,BR16,BR20,BR15,BR27,BR4

في ثلاثه مو اقع مختلفة هي المزر عه البحثيه بقها - محافظه القليوبيه وتروى بماء عذب بينما الموقعين الأخريين تابعين

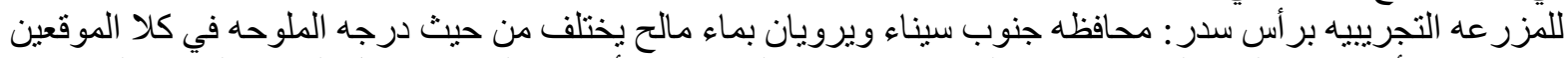

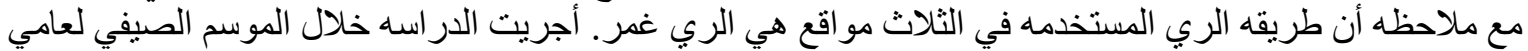

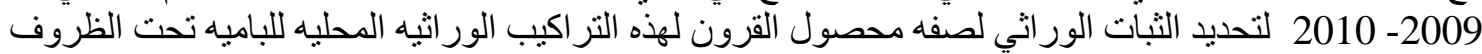
Additive Main Effect and Multiplicative المختلفه من درجه الملوحه ـ أستخدم نموذج تحليل الثبات الوراثي Interaction Analysis Interactive principle component axis 1,axis2,.......etc.(IPCA1,IPCA2,IPCA3,....etc). كفاءه من الطرق التقليديه الأخري مثل مقياس الإنحدار(بناء على أبحاث متعدده) و الذي يعتمد على الإنحر اف الف المعياري.

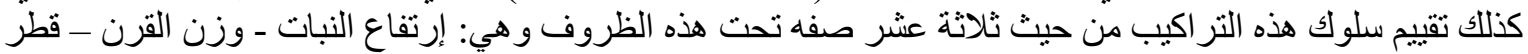

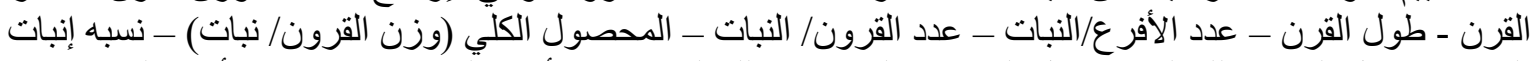
البذور - طول الجذر ( للثتله) - طول المجموع الخزون التضري (للشتله)- تركيز أيون الصوديوم - تركيز أيون البوناسيوم -

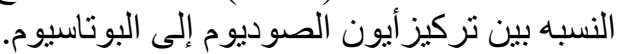
وكانت أهم النتائج المتحصل أنسئ عليها كالأتي:-

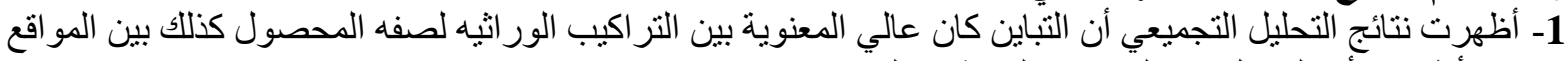

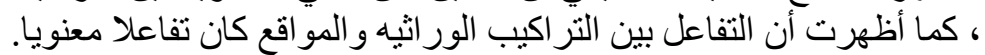

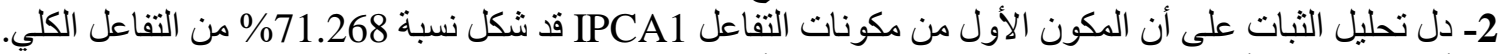

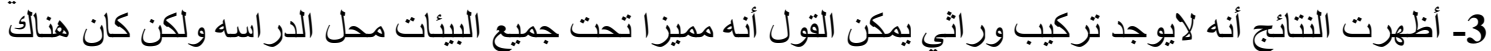

تر اكيب أكثر ملائمه لمدى واسع من الظروف لائر الملحيه وهي:BG9,BG6 BR27 and BR20

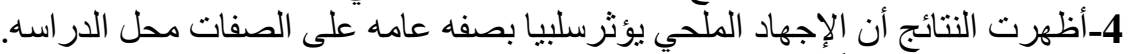

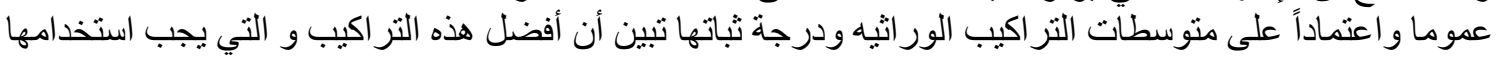

كآباء في بر امج التربية و تطوير صفة التحمل للملوحه هي:BG9,BG6 BR27 and BR20

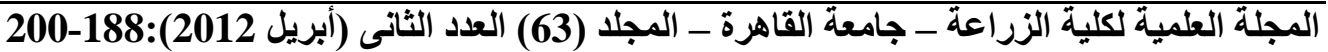

